\title{
1 A critical period terminates the differentiation of olfactory sensory neurons
}

2

3 Shadi Jafari $1^{1,2}$ and Mattias Alenius ${ }^{1,3}$

4

5 1. Department of Clinical and Experimental Medicine, Linköping University,

6 Linköping, Sweden

7 2. Current affiliation: Department of Biology, New York University, New York, USA

8 3. Current affiliation: Department of Molecular Biology, Umeå University, Umeå, SE 901 87,

9 Sweden

10

11

12

13

14

15

16

17

18

19

20

21

22

23

24 
25

26

27

28

29

\section{Abstract}

Development generates a vast number of neuron types and classes. When and how neuronal differentiation end is poorly understood. Here, we show that Drosophila olfactory sensory neurons (OSNs) matures during a critical period and reveal that the differentiation termination mechanism is similar to the mammalian odorant receptor $(\mathrm{OR})$ choice mechanism. We first showed that initiation of Drosophila OR expression required heterochromatin opening and a H3K9me3 demethylase, $K d m 4 b$. Further genetic studies demonstrated that $L s d 1$ and $s u(v a r) 3-$ 9, similar to mouse, were required to balance heterochromatin in order to stabilize OR expression. Expression analysis showed that $L s d 1$, su(var)3-9 increased and $K d m 4 b$ decreased during the first two days after eclosion. We further showed that environment changes during the period, but not after, caused permanent transformed Lsd1, su(var)3-9 and Kdm4b expression and altered OR gene regulation. These results together suggest the last step in OSN terminal differentiation to be a gene regulatory critical period. 


\section{Introduction}

OSNs in most vertebrates and insects get specified to express a single odorant receptor (OR) from a large repertoire of OR genes in the genome and generate classes of OSNs that express a certain OR (Couto, Alenius, \& Dickson, 2005; Fishilevich \& Vosshall, 2005; Mombaerts et al., 1996; Ressler, Sullivan, \& Buck, 1994). In Drosophila, it is assumed that the monogenic OR gene expression is strictly predetermined and non-stochastic (Barish \& Volkan, 2015; Jafari et al., 2012; Ray, van Naters, \& Carlson, 2008). There are several reasons for this assumption. For one, OR expression is stereotyped organised (Couto et al., 2005). Drosophila OSNs are also specified in a lineage dependent manner (Barish \& Volkan, 2015) that is dictated by Notch and divide the post mitotic OSNs into two subgroups with defined projection patterns and expressed ORs (Endo, Aoki, Yoda, Kimura, \& Hama, 2007). In addition to Notch and its effects, combinations of at least ten transcription factors (TFs) are required to express the different ORs (Jafari et al., 2012; Komiyama, Carlson, \& Luo, 2004; Sim, Perry, Tharadra, Lipsick, \& Ray, 2012; Tichy, Ray, \& Carlson, 2008). However, while necessary, each TF combination is not sufficient to specify OR expression, as the defined TF combination is found elsewhere in the nervous system where the OR is not expressed (Jafari et al., 2012), suggesting that additional mechanisms restrict Drosophila OR expression.

Conversely, OR expression in vertebrates is considered to be less strict regulated and in part stochastic (Abdus-Saboor, Fleischmann, \& Shykind, 2014; Monahan \& Lomvardas, 2015). Cyclical changes in chromatin state from 'active' to 'repressed' and back again have been acknowledged as means for regulation of OR expression (Lyons et al., 2013; Magklara et al., 2011). In mouse and zebrafish, OR genes are embedded in constitutive heterochromatin in immature OSNs and in most non-olfactory tissues (Magklara et al., 2011). Constitutive heterochromatin is a condensed and transcriptionally inert chromatin conformation, marked by histone $\mathrm{H} 3$ lysine 9 trimethylation (H3K9me3) (Filion et al., 2010). Mathematical modelling 
of the vertebrate OR choice propose that the opening of constitutive heterochromatin around the OR gene initiate expression (Tan, Zong, \& Xie, 2013) and requires a yet-to-be-identified H3K9me3 demethylase (Lyons et al., 2013). Next, Lsd1 remove the methyl groups from the OR locus marked with $\mathrm{H} 3 \mathrm{~K} 9 \mathrm{me} 2$, which further open the chromatin and secure the OR expression initiation (Lyons et al., 2013). The folding or activity of the expressed OR induce several feedback loops that suppress other OR genes expression through down regulation of Lsd1 or upregulation of heterochromatin formation (Dalton, Lyons, \& Lomvardas, 2013; Ferreira et al., 2014; Fleischmann, Abdus-Saboor, Sayed, \& Shykind, 2013; Lyons et al., 2013).

Drosophila OR promoters are enriched with the H3K9me2 mark (Sim et al., 2012). Sim et al also show that su(var)3-9, which produce $\mathrm{H} 3 \mathrm{~K} 9 \mathrm{me} 3$ and form constitutive heterochromatin, suppress spurious OR expression in Drosophila. Thermodynamic modeling based on detailed genetic studies of Or59b gene regulation put forward that cooperative interactions between transcription factors defy $\mathrm{Su}(\mathrm{var}) 3-9$ suppression and promote Drosophila OR expression (Gonzalez, Jafari, Zenere, Alenius, \& Altafini, 2019; Jafari \& Alenius, 2015).

Here, we further address the role of heterochromatin in Drosophila OR regulation and show that similar to vertebrates, $s u(v a r) 3-9$ and $d L s d 1$ are a key factors in the commencement and maintenance of restricted OR expression. We reveal that the H3K9me3 demethylase, $K d m 4 b$, is required to initiate Drosophila OR expression. We demonstrate that $\mathrm{Kdm} 4 \mathrm{~b}$, $\mathrm{dLsd} 1$ and su(var)3-9 regulation is part of the closing mechanism of a gene regulatory critical period that marks the final step in the Drosophila OSN differentiation. 
97

\section{Results}

\section{$K d m 4 b$ initiate OR expression}

Vertebrate OR expression is hypothesized to be initiated by an unknown factor that opens the constitutive heterochromatin at a single OR locus (Lyons et al., 2013; Tan et al., 2013). Our previous results demonstrate that whilst $O r 59 \mathrm{~b}$ expression is stabilized by a constitutive heterochromatin configuration (Jafari \& Alenius, 2015), computer modeling recently predicted that its opening is required for Or59b expression (Gonzalez et al., 2019). This event is initiated by H3K9me3 demethylation (Klose et al., 2006). In Drosophila, there are two H3K9me3 demethylases, $K d m 4 a$ (Kdm4B in vertebrates) and $K d m 4 b$ (Kdm4A, C, D, E in vertebrates) (Greer \& Shi, 2012). UAS-IR (inverted repeats) for the two demethylases was expressed with Peb-Gal4 in the OSNs and the knock down of the two demethylases showed that $K d m 4 b$ but not $K d m 4 a$ is required for $O r 59 b$ expression (Figure 1A). The two Kdm4 enzymes have affinity for both $\mathrm{H} 3 \mathrm{~K} 9$ and H3K36 but Kdm4b is the major H3K9 demethylase (Tsurumi, Dutta, Shang, Yan, \& Li, 2013), supporting that opening of heterochromatin initiate Or59b expression. To address if $\mathrm{Kdm} 4 \mathrm{~b}$ is required for continuous $O r 59 \mathrm{~b}$ expression, we knocked down $\mathrm{Kdm} 4 \mathrm{~b}$ after OR initiation. As initiation of OR expression starts in the mid-pupal stages, we utilized OrcoGal4 which is expressed immediately before hatching (Larsson et al., 2004). Orco-Gal4 driven $\mathrm{Kdm} 4 \mathrm{~b}$ knock down produced no changes in Or59b expression (Figure 1B), which indicate that $\mathrm{Kdm} 4 \mathrm{~b}$ is only required to initiate $\mathrm{Or} 59 \mathrm{~b}$ expression.

\section{$d L s d 1$ and $S u(v a r) 3-9$ balance OR expression}

Kdm4b converts H3K9me3 to H3K9me2 (Tsurumi et al., 2013). H3K9me2 in mouse OSNs is the substrate for Lsd1, which catalyse further demethylation to H3K9 and induce stable OR expression (Lyons et al., 2013). To investigate if dLsd1 is also required for Drosophila OR expression, we knocked down $d L s d 1$ in OSNs with Peb-Gal4. Or59b expression was lost in 
the knock down flies (Figure 2A). Analysis of two additional ORs showed that whilst Or92a expression was completely abolished in knock down antennae (Figure 2B, VA2), no change was observed for $O r 47 b$ expression (Figure 2B, Valv), demonstrating that dLsd1 is required for the expression of some but not all ORs.

During Drosophila development, dLsd1 promotes closing of heterochromatin, and not its opening (Di Stefano, Ji, Moon, Herr, \& Dyson, 2007; Rudolph et al., 2007). In both Drosophila and mouse, heterochromatin formation is regulated by $S u(v a r) 3-9$, which catalyses the methylation of H3K9me2 to H3K9me3 (Nakayama, Rice, Strahl, Allis, \& Grewal, 2001; Rea et al., 2000; Schotta et al., 2002). To address if dLsd1 counteracts $\operatorname{Su(var)3-9~activity~during~}$ Or59b gene regulation, we reduced the gene copy number of either $d L s d 1$ or $S u(v a r) 3-9$, or both. To sensitize the analysis we used a Or59b minimal enhancer reporter (Or59bME) that lack the cooperative transcription factor interactions required to defy heterochromatin formation (Jafari \& Alenius, 2015). Or59bME reporter is expressed in the Or59b OSN class that project to the DM4 glomerulus but the reporter expression is sensitive to changes in chromatin state (Figure 2C (Jafari \& Alenius, 2015). Consistently, in both $d L s d 1$ and Su(var)39 heterozygote flies Or59bME reporter expression was lost (Figure 2C). Interestingly, combining the two heterozygotes rescued the single class expression of the reporter (Figure 2D). That restoring the copy number of the two genes complete rescued Or59bME regulation supports a model where dLsd1 opposes $\mathrm{Su}(\mathrm{var})$ 3-9 and promotes open heterochromatin in Drosophila OSNs. The results further demonstrate that strict coordination and balance of dLsd1 and $\mathrm{Su}(\mathrm{var}) 3-9$ levels or function is required to support $\mathrm{Or} 59 \mathrm{~b}$ expression. Knock down after OR initiation of $d L s d 1$ resulted in loss of $O r 59 b M E$ expression, but not that of $O r 59 \mathrm{~b}$ itself (Figure 2E), supporting that $d L s d 1$ continuously counter act heterochromatin formation and that transcription factor cooperativity reduce its requirement in the adult state. Interestingly, Lhx2, one of few transcription factors known to regulate vertebrate OR expression (Kolterud, 
146 Alenius, Carlsson, \& Bohm, 2004), also requires cooperativity to counteract local H3K9me3 and heterochromatin formation in the OSNs (Monahan et al., 2017).

\section{HP1b and dLbr is required for Or59b expression}

Heterochromatin on a local level represses expression and on a global level function together with HP1 proteins as attachment sites for the inner nuclear membrane anchor proteins that compartmentalize the nucleus into hetero- and eu-chromatin domains (van Steensel \& Belmont, 2017; Wong, Luperchio, \& Reddy, 2014). Nuclear localization of the vertebrate OR loci require the lamin b receptor (Lbr1) (Clowney et al., 2012). Knock down of Drosophila $d L b r 1$ in OSNs by Peb-Gal4 drastically reduced both Or59b and minimal enhancer reporter expression (Figure 3A, B). Lbr1 interacts with HP1 proteins (Polioudaki et al., 2001; Ye \& Worman, 1996). Knock down of HP1b but not HP1a, HP1c nor HP1d caused loss of Or59b expression (Figure 3B and Figure S1). Expression of the minimal enhancer reporter was also lost in both dLbr1 and HP1b knock down flies (Figure 3A). The Or59b reporter and the minimal enhancer reporter are inserted at different locations in the genome, which suggest thatthe OR reporters instructed the HP1 and dLbr1 function independent of locus location. Dynamic Kdm4b, dLsd1 and Su(var)3-9 expression modulate Or59b expression.

In immature flies, Or $59 \mathrm{~b}$ expression varied in level between OSNs and during the first days matured to the stable uniform expression level observed in adults (Figure 4A). Quantitative PCR (qPCR) experiments showed that $d L s d 1$ and $S u(v a r) 3-9$ expression increased in the antennae during the first days post eclosion (DPE), peaking in three-day old flies (Figure 4B), correlating with the maturation in OR expression. A more detailed analysis showed that the main increase in expression occurred during the first hours post eclosion (Figure 4C), which suggests that early exposure to odors and other environmental cues can modulate $d L s d l$ and 
and remained low in adult flies (Figure 4D) further corroborates the idea that OR initiation and expression permissiveness decrease during a restricted time in early Drosophila life.

To address if $d L s d 1$ and/or $S u(v a r) 3-9$ expression also change in the Drosophila central nervous system, we performed qPCR on cDNA from brains of newly eclosed, three and sevenday old Drosophila. Interestingly, during the first three days $d L s d 1$ expression increased to the adult level (Figure 4E), showing that a similar general shift in $d L s d 1$ expression occurs in the brain. Surprisingly, Su(var)3-9 expression was not altered (Figure 4E), indicating that the mechanism and change in gene regulation in the brain is different to the OSNs.

\section{$d L s d 1$ and $s u(v a r) 3-9$ expression changes are a part of an OSN gene regulatory critical period}

The well-defined and restricted temporal window of the refinement in OR expression (figure 4A) and change of $s u(v a r) 3-9, d L s d 1$ and $K d m 4 b$ expression resemble a critical period. If the observed changes is part of a critical period, the system should be sensitized to changes in the environment and neuronal activity during this time (Hensch, 2004). To address if the observed changes in $d L s d 1$ and $S u(v a r) 3-9$ expression is sensitive to environmental cues, we analysed changes to their levels in flies shifted from ambient to low temperature at different time points

(Figure 5A). Flies subjected to a temperature shift at eclosion (1 DPE) showed a twofold reduction of $d L s d 1$ and $S u($ var $)$ 3-9 expression (Figure 5B). Interestingly, a similar shift in adult flies (7 DPE) did not affect $S u(v a r) 3-9$ expression but $d L s d 1$ expression dropped to the level found at eclosion (Figure 5B). Thus, thermal stress differentially regulates $d L s d 1$ and $S u(v a r) 3-$ 9 expression in immature and adult flies, supporting the notion that modulation of $d L s d 1$ and $S u(v a r) 3-9$ expression takes place during a critical period of OSN gene regulation.

To visualize the effect of these events on OR gene regulation, we returned to the Or59bME reporter. At ambient temperature the Or59bME reporter was expressed in the Or59b OSN class 
that projects to the DM4 glomerulus (Figure 5C). Or59bME reporter flies shifted to low temperature immediately after eclosion showed a marked ectopic reporter expression with several GFP positive glomeruli (Figure 5C), which is consistent with the observed lowered Su(var)3-9 and $d L s d 1$ expression (Figure 5B). Temperature shifts in adult flies produced mainly loss of expression (Figure 5C), which also tallies in with the observed imbalance in Su(var)3-9 and $d L s d 1$ expression (figure 5B). Shorter shifts to low temperatures showed that the switch in reporter expression regulation occurred between day 2 and day 3 post eclosion (Figure 5D, E). Altogether, these results showed that there is a critical period in OSN gene regulation and that it is limited to the first two days' post eclosion.

\section{Temperature stress during the critical period induce permanent changes in OSN gene}

\section{expression}

According to the defining criteria of a critical period, the state of the system at the end of the period should be permanent and irreversible (Hensch, 2004). To investigate if this is the case for $\operatorname{Or} 59 \mathrm{bME}$ regulation, newly eclosed reporter flies were shifted to low temperature for three days and then returned to the ambient temperature for seven days. Consistent with a permanent change, the ectopic Or59bME reporter expression pattern persisted the seven-day recovery period (Figure 6A). Even after a prolonged 18-day recovery period the Or59bME expression pattern was comparable with that observed directly after the intervention (Figure 6A), demonstrating that the change in gene regulation during the first days was irreversible.

If the critical period induces a transcriptional memory, the loss of Or59bME reporter expression observed after an adult temperature shift should recover to the expression pattern at the end of the critical period. Consistently, if 7 day old Or59bME reporter flies were shifted to low temperatures for three days, the loss of expression recovered to single OSN class expression (Figure 6B), demonstrating that temperature shifts after the critical period have only 
transient effects on the Or59bME reporter expression. To test if the critical period in fact produces a transcriptional memory and an irreversible permanent state, we challenged the ectopic expression of an early shift by a second adult shift to the same low temperature conditions. Strikingly, the ectopic expression pattern of the ME reporter was comparable between flies subjected to one or two shifts (Figure 6A, B). This demonstrated that the temperature during the critical period dictated $\operatorname{Or} 59 \mathrm{bME}$ expression pattern in adult, thus producing a lasting transcriptional memory.

\section{Discussion}

Here, we demonstrate that OSNs transition from a deterministic differentiation that produce variable OR expression levels to the mature stable high OR expression level observed in adult flies during a short window early in Drosophila life. This transition has the hallmarks of a critical period (Hensch, 2004) with a sharply-defined temporal window, sensitivity to external modulation, in this case environmental stress and generation of a permanent adult state, a terminal differentiated neuron.

Our findings further demonstrate that OSN differentiation during the critical period utilizes a mechanism similar to vertebrate OR choice. We first show that, as predicted for vertebrate OR expression (Tan et al., 2013), a H3K9me3 demethylase, Kdm4b initiates Drosophila OR expression. We further show that similar to the role in vertebrate OR expression, dLsd1 establish ORs expression in Drosophila. Our results and several vertebrate OR choice studies (Coleman, Lin, \& Schwob, 2017; Lyons et al., 2013; Vyas, Meredith, \& Lane, 2017) show that in the OSNs Lsd1 act as an activator that opposes $\mathrm{Su}(\mathrm{var}) 3-9$ and constitutive heterochromatin formation. In most other Drosophila and vertebrate cells, Lsd1 function as a repressor that demethylates H3K4me1/2 and induces heterochromatin formation (Di Stefano et al., 2007; Rudolph et al., 2007), indicating that the distinct Lsd1 function in OSNs is a conserved feature. 
The criteria for a critical period (Hensch, 2004) suggest that neuronal activity and competition is also part of the Drosophila OR regulation. Interestingly, there is evidence for competition between ORs in Drosophila (Shaw, Johnson, Anderson, de Bruyne, \& Warr, 2019). Or22a and Or22b are two ORs expressed in a single class (Couto et al., 2005). In the wild Drosophila melanogaster express either Or22a or Or $22 \mathrm{~b}$ but not both, suggesting that there is an active suppression mechanism that maintain monogenic expression. In immature vertebrate OSNs, there is a low frequency of OR co-expression and biallelic expression (Hanchate et al., 2015; Shykind et al., 2004). To secure monogenic OR expression several feedback loops later suppress the spurious OR expression and restrict expression to the first or perhaps most expressed OR (Abdus-Saboor et al., 2014). Refinement feedback mechanisms build on that folding (Dalton et al., 2013; Lyons et al., 2013) or activity of the expressed OR (Ferreira et al., 2014; Fleischmann et al., 2013) suppress other OR genes expression through down regulation of Lsd1 or upregulation of heterochromatin formation. Thus, it will be of great interest to investigate if there are feedback mechanisms that link Drosophila OR expression or OSN activity with $S u(v a r) 3-9$ and $d L s d 1$ expression and regulate the critical period.

After its initiation, OR expression regulation differs between Drosophila and mouse. The most dramatic difference is that mouse ORs are mono allelic expressed whereas Drosophila ORs are like most genes biallelic expressed. Our results show that continuous Drosophila OR expression requires both $d L s d l$ and $S u(v a r) 3-9$ and that heterochromatin state is under constant regulation, which underscore the difference to vertebrates where Lsd1 is down regulated after the initiation of the mono allelic expression. Together with the discovered critical period the constant regulation of heterochromatin implies that there must be a transcription memory mechanism, which sustain continuous OR expression. Our results together with recent published results indicate that nuclear organisation can be part of such a memory mechanism. 
267 (Polioudaki et al., 2001; Ye \& Worman, 1996) and recent ChIP experiments show that in Drosophila neurons actively transcribed gene regions have a HP1/H3K9me2 profile (Pindyurin et al., 2018). A model where HP1 attach H3K9me2 heterochromatin with LBR and like that stabilize the nuclear organization of the OSN and OR expression could thus explain the somewhat surprising requirement for heterochromatin in Or59b expression. The need to coordinate $\mathrm{H} 3 \mathrm{~K} 9 \mathrm{me} 2$ and heterochromatin levels is further underscored by that stable OR expression require the $\mathrm{H} 3 \mathrm{~K} 9 \mathrm{me} 2$ methylase G9a in both mouse and Drosophila (Alkhori, Ost,

\& Alenius, 2014; Lyons et al., 2014).

Our qPCR results reveal an increase in $d L s d 1$ expression during the first days in the brain. A change in $\mathrm{H} 3 \mathrm{~K} 9 \mathrm{me} 2$ regulation can thus be part of a potential general critical period in the Drosophila brain. Interestingly, there is no change in $S u(v a r) 3-9$ expression, which suggest that in early-life Drosophila the contrast in gene regulation between central neurons and OSNs increase. Thus, one direct implication of the lack of $S u(v a r) 3-9$ change is that it might suppress

OR expression in the central nervous system, which would be an elegant and simple solution to limit OR expression to the OSNs. Still, if there is a general critical period in terminal differentiation of Drosophila neurons and how $d L s d 1$ and $S u(v a r) 3-9$ expression is regulated in central and sensory neurons are interesting questions that remains to be addressed.

Materials and Methods

\section{Drosophila stocks}

Or59b promoter fusion and Or59b minimal enhancer constructs have previously been described

(Couto et al., 2005; Jafari \& Alenius, 2015). The Pebbled-Gal4 (Peb-Gal4) was a kind gift from Liqun Luo (Stanford University, Stanford, CA, USA). The $s u(v a r) 3-9^{06}$ and $L s d 1^{09}$ mutants were a kind gift from Anita Öst (Linköping University, Linköping, Sweden). The following fly lines were provided by the Bloomington Drosophila Stock Center: $w^{1118}(38690)$, 
Orco-Gal4 (23909), su(var)2055 (12226) dLsd1-IR (36867; 32853, 33726), dLbr-IR (53269),

Flies were raised and maintained on standard Drosophila culture medium at $24^{\circ} \mathrm{C}$ and collected

transferred to new vials and maintained for 3 days at $14^{\circ} \mathrm{C}$.

297

298

299

300

301

302

303

304

305

306

307

\section{Immunofluorescence}

Immunofluorescence was performed according to previously described methods. The following primary antibodies were used: rabbit anti-GFP (1:2000, TP-401; Torrey Pines Biolabs) and mouse anti-nc82 (1:100; DSHB). Secondary antibodies were conjugated with Alexa Fluor 488 (1:500; Molecular Probes) and Goat anti-Mouse IgG (H+L) Cross-Adsorbed Secondary Antibody, Rhodamine Red-X (1:250), Thermo Fisher). Confocal microscopy images were collected on an LSM 700 (Zeiss) and analyzed using an LSM Image Browser. Adobe Photoshop CS4 (Adobe Systems) was used for image processing.

\section{qPCR}

After freezing flies in liquid nitrogen antennae were obtained with a sieve. Total RNA from antennae was extracted with TRIzol reagent (Invitrogen) and the RNeasy kit (Qiagen). Quantitative PCR was conducted with an Applied Biosystems 7900HT real-time PCR system (Life Technologies) using the Power SYBR Green PCR master mix (Applied Biosystems, Life Technologies) and primer sets designed using Primer Express software v3.0.1 (Integrated DNA Technologies). Tubulin and Actin were used as an internal control for the experiments. To amplify cDNA products and not genomic DNA, primers were designed to join the end of one exon with the beginning of the next exon. Quantitative PCR for each primer set was performed on both control and experimental samples for 40 cycles. Following amplification, melt curve 
analysis and ethidium bromide agarose gel electrophoresis were performed to evaluate the PCR products. The relative quantification of the fold change in mRNA expression was calculated using the $2-\Delta \Delta \mathrm{CT}$ threshold cycle method.

\section{Acknowledgements}

We thank, Anita Öst for flies; Najat Dzaki, Carlos Ribeiro and Staffan Bohm for discussions and comments on the manuscript. This work was supported by the Swedish Research foundation, grant (2016-05208)

\section{References}

Abdus-Saboor, I., Fleischmann, A., \& Shykind, B. (2014). Setting Limits: Maintaining order in a large gene family. Transcription, 5 . Retrieved from http://www.ncbi.nlm.nih.gov/pubmed/24802893

Alkhori, L., Ost, A., \& Alenius, M. (2014). The corepressor Atrophin specifies odorant receptor expression in Drosophila. Faseb Journal, 28(3), 1355-1364. doi:10.1096/fj.13-240325

Barish, S., \& Volkan, P. C. (2015). Mechanisms of olfactory receptor neuron specification in Drosophila. Wiley Interdisciplinary Reviews-Developmental Biology, 4(6), 609-621. doi:10.1002/wdev.197

Clowney, E. J., LeGros, M. A., Mosley, C. P., Clowney, F. G., Markenskoff-Papadimitriou, E. C., Myllys, M., . . Lomvardas, S. (2012). Nuclear aggregation of olfactory receptor genes governs their monogenic expression. Cell, 151(4), 724-737. doi:10.1016/j.cell.2012.09.043

Coleman, J. H., Lin, B., \& Schwob, J. E. (2017). Dissecting LSD1-Dependent Neuronal Maturation in the Olfactory Epithelium. J Comp Neurol, 525(16), 3391-3413. doi:10.1002/cne.24259

Couto, A., Alenius, M., \& Dickson, B. J. (2005). Molecular, anatomical, and functional organization of the Drosophila olfactory system. Current Biology, 15(17), 1535-1547. doi:S09609822(05)00789-X [pii]

10.1016/j.cub.2005.07.034

Dalton, R. P., Lyons, D. B., \& Lomvardas, S. (2013). Co-opting the unfolded protein response to elicit olfactory receptor feedback. Cell, 155(2), 321-332. doi:10.1016/j.cell.2013.09.033

Di Stefano, L., Ji, J. Y., Moon, N. S., Herr, A., \& Dyson, N. (2007). Mutation of Drosophila Lsd1 disrupts H3-K4 methylation, resulting in tissue-specific defects during development. Current Biology, 17(9), 808-812. doi:10.1016/j.cub.2007.03.068

Endo, K., Aoki, T., Yoda, Y., Kimura, K., \& Hama, C. (2007). Notch signal organizes the Drosophila olfactory circuitry by diversifying the sensory neuronal lineages. Nat Neurosci, 10(2), 153160. doi:nn1832 [pii]

$10.1038 / \mathrm{nn} 1832$

Ferreira, T., Wilson, S. R., Choi, Y. G., Risso, D., Dudoit, S., Speed, T. P., \& Ngai, J. (2014). Silencing of odorant receptor genes by $\mathrm{G}$ protein betagamma signaling ensures the expression of one 
odorant receptor per olfactory sensory neuron. Neuron, 81(4), 847-859. doi:10.1016/j.neuron.2014.01.001

Filion, G. J., van Bemmel, J. G., Braunschweig, U., Talhout, W., Kind, J., Ward, L. D., . . van Steensel, B. (2010). Systematic protein location mapping reveals five principal chromatin types in Drosophila cells. Cell, 143(2), 212-224. doi:10.1016/j.cell.2010.09.009

Fishilevich, E., \& Vosshall, L. B. (2005). Genetic and functional subdivision of the Drosophila antennal lobe. Current Biology, 15(17), 1548-1553. doi:S0960-9822(05)00893-6 [pii]

10.1016/j.cub.2005.07.066

Fleischmann, A., Abdus-Saboor, I., Sayed, A., \& Shykind, B. (2013). Functional interrogation of an odorant receptor locus reveals multiple axes of transcriptional regulation. Plos Biology, 11(5), e1001568. doi:10.1371/journal.pbio.1001568

Gonzalez, A., Jafari, S., Zenere, A., Alenius, M., \& Altafini, C. (2019). Thermodynamic model of gene regulation for the Or59b olfactory receptor in Drosophila. PLoS Comput Biol, 15(1), e1006709. doi:10.1371/journal.pcbi.1006709

Greer, E. L., \& Shi, Y. (2012). Histone methylation: a dynamic mark in health, disease and inheritance. Nat Rev Genet, 13(5), 343-357. doi:10.1038/nrg3173

Hanchate, N. K., Kondoh, K., Lu, Z., Kuang, D., Ye, X., Qiu, X., . . Buck, L. B. (2015). Single-cell transcriptomics reveals receptor transformations during olfactory neurogenesis. Science, 350(6265), 1251-1255. doi:10.1126/science.aad2456

Hensch, T. K. (2004). Critical period regulation. Annual Review of Neuroscience, 27, 549-579. doi:10.1146/annurev.neuro.27.070203.144327

Jafari, S., \& Alenius, M. (2015). Cis-Regulatory Mechanisms for Robust Olfactory Sensory Neuron Class-restricted Odorant Receptor Gene Expression in Drosophila. Plos Genetics, 11(3). doi:ARTN e1005051

\subsection{1/journal.pgen.1005051}

Jafari, S., Alkhori, L., Schleiffer, A., Brochtrup, A., Hummel, T., \& Alenius, M. (2012). Combinatorial activation and repression by seven transcription factors specify Drosophila odorant receptor expression. Plos Biology, 10(3), e1001280. doi:10.1371/journal.pbio.1001280

Klose, R. J., Yamane, K., Bae, Y., Zhang, D., Erdjument-Bromage, H., Tempst, P., . . Zhang, Y. (2006). The transcriptional repressor JHDM3A demethylates trimethyl histone $\mathrm{H} 3$ lysine 9 and lysine 36. Nature, 442 (7100), 312-316. doi:10.1038/nature04853

Kolterud, A., Alenius, M., Carlsson, L., \& Bohm, S. (2004). The Lim homeobox gene Lhx2 is required for olfactory sensory neuron identity. Development, 131(21), 5319-5326. doi:10.1242/dev.01416

dev.01416 [pii]

Komiyama, T., Carlson, J. R., \& Luo, L. (2004). Olfactory receptor neuron axon targeting: intrinsic transcriptional control and hierarchical interactions. Nat Neurosci, 7(8), 819-825. doi:10.1038/nn1284

nn1284 [pii]

Larsson, M. C., Domingos, A. I., Jones, W. D., Chiappe, M. E., Amrein, H., \& Vosshall, L. B. (2004). Or83b encodes a broadly expressed odorant receptor essential for Drosophila olfaction. Neuron, 43(5), 703-714. doi:10.1016/j.neuron.2004.08.019

Lyons, D. B., Allen, W. E., Goh, T., Tsai, L., Barnea, G., \& Lomvardas, S. (2013). An epigenetic trap stabilizes singular olfactory receptor expression. Cell, 154(2), 325-336. doi:10.1016/j.cell.2013.06.039

Lyons, D. B., Magklara, A., Goh, T., Sampath, S. C., Schaefer, A., Schotta, G., \& Lomvardas, S. (2014). Heterochromatin-mediated gene silencing facilitates the diversification of olfactory neurons. Cell Rep, 9(3), 884-892. doi:10.1016/j.celrep.2014.10.001 
bioRxiv preprint doi: https://doi.org/10.1101/2020.02.12.945428; this version posted February $12,2020$. The copyright holder for this preprint (which was not certified by peer review) is the author/funder, who has granted bioRxiv a license to display the preprint in perpetuity. It is made available under aCC-BY 4.0 International license.

Magklara, A., Yen, A., Colquitt, B. M., Clowney, E. J., Allen, W., Markenscoff-Papadimitriou, E., ... Lomvardas, S. (2011). An epigenetic signature for monoallelic olfactory receptor expression. Cell, 145(4), 555-570. doi:10.1016/j.cell.2011.03.040

Mombaerts, P., Wang, F., Dulac, C., Chao, S. K., Nemes, A., Mendelsohn, M., . . Axel, R. (1996). Visualizing an olfactory sensory map. Cell, 87(4), 675-686. doi:S0092-8674(00)81387-2 [pii]

Monahan, K., \& Lomvardas, S. (2015). Monoallelic expression of olfactory receptors. Annu Rev Cell Dev Biol, 31, 721-740. doi:10.1146/annurev-cellbio-100814-125308

Monahan, K., Schieren, I., Cheung, J., Mumbey-Wafula, A., Monuki, E. S., \& Lomvardas, S. (2017). Cooperative interactions enable singular olfactory receptor expression in mouse olfactory neurons. Elife, 6. doi:10.7554/eLife.28620

Nakayama, J., Rice, J. C., Strahl, B. D., Allis, C. D., \& Grewal, S. I. (2001). Role of histone H3 lysine 9 methylation in epigenetic control of heterochromatin assembly. Science, 292(5514), 110113. doi:10.1126/science. 1060118

Pindyurin, A. V., Ilyin, A. A., Ivankin, A. V., Tselebrovsky, M. V., Nenasheva, V. V., Mikhaleva, E. A., . . Shevelyov, Y. Y. (2018). The large fraction of heterochromatin in Drosophila neurons is bound by both B-type lamin and HP1a. Epigenetics Chromatin, 11(1), 65. doi:10.1186/s13072-018-0235-8

Polioudaki, H., Kourmouli, N., Drosou, V., Bakou, A., Theodoropoulos, P. A., Singh, P. B., .. . Georgatos, S. D. (2001). Histones H3/H4 form a tight complex with the inner nuclear membrane protein LBR and heterochromatin protein 1. EMBO Rep, 2(10), 920-925. doi:10.1093/embo-reports/kve199

Ray, A., van Naters, W. V., \& Carlson, J. R. (2008). A regulatory code for neuron-specific odor receptor expression. Plos Biology, 6(5), 1069-1083. doi:ARTN e125

\subsection{1/journal.pbio.0060125}

Rea, S., Eisenhaber, F., O'Carroll, D., Strahl, B. D., Sun, Z. W., Schmid, M., ... Jenuwein, T. (2000). Regulation of chromatin structure by site-specific histone $\mathrm{H} 3$ methyltransferases. Nature, 406(6796), 593-599. doi:10.1038/35020506

Ressler, K. J., Sullivan, S. L., \& Buck, L. B. (1994). Information coding in the olfactory system: evidence for a stereotyped and highly organized epitope map in the olfactory bulb. Cell, 79(7), 12451255. doi:0092-8674(94)90015-9 [pii]

Rudolph, T., Yonezawa, M., Lein, S., Heidrich, K., Kubicek, S., Schafer, C., . . Reuter, G. (2007). Heterochromatin formation in Drosophila is initiated through active removal of H3K4 methylation by the LSD1 homolog SU(VAR)3-3. Mol Cell, 26(1), 103-115. doi:10.1016/j.molcel.2007.02.025

Schotta, G., Ebert, A., Krauss, V., Fischer, A., Hoffmann, J., Rea, S., . . Reuter, G. (2002). Central role of Drosophila SU(VAR)3-9 in histone H3-K9 methylation and heterochromatic gene silencing. EMBO J, 21(5), 1121-1131. doi:10.1093/emboj/21.5.1121

Shaw, K. H., Johnson, T. K., Anderson, A., de Bruyne, M., \& Warr, C. G. (2019). Molecular and Functional Evolution at the Odorant Receptor Or22 Locus in Drosophila melanogaster. Mol Biol Evol, 36(5), 919-929. doi:10.1093/molbev/msz018

Shykind, B. M., Rohani, S. C., O'Donnell, S., Nemes, A., Mendelsohn, M., Sun, Y., . . Barnea, G. (2004). Gene switching and the stability of odorant receptor gene choice. Cell, 117(6), 801815. doi:10.1016/j.cell.2004.05.015

\section{S009286740400529X [pii]}

Sim, C. K., Perry, S., Tharadra, S. K., Lipsick, J. S., \& Ray, A. (2012). Epigenetic regulation of olfactory receptor gene expression by the Myb-MuvB/dREAM complex. Genes Dev, 26(22), 24832498. doi:10.1101/gad.201665.112

Tan, L. Z., Zong, C. H., \& Xie, X. S. (2013). Rare event of histone demethylation can initiate singular gene expression of olfactory receptors. Proceedings of the National Academy of Sciences of the United States of America, 110(52), 21148-21152. doi:10.1073/pnas.1321511111 
Tichy, A. L., Ray, A., \& Carlson, J. R. (2008). A new Drosophila POU gene, pdm3, acts in odor receptor expression and axon targeting of olfactory neurons. Journal of Neuroscience, 28(28), 71217129. doi:28/28/7121 [pii]

\subsection{3/JNEUROSCI.2063-08.2008}

Tsurumi, A., Dutta, P., Shang, R., Yan, S. J., \& Li, W. X. (2013). Drosophila Kdm4 demethylases in histone H3 lysine 9 demethylation and ecdysteroid signaling. Sci Rep, 3, 2894. doi:10.1038/srep02894

van Steensel, B., \& Belmont, A. S. (2017). Lamina-Associated Domains: Links with Chromosome Architecture, Heterochromatin, and Gene Repression. Cell, 169(5), 780-791. doi:10.1016/j.cell.2017.04.022

Vyas, R. N., Meredith, D., \& Lane, R. P. (2017). Lysine-specific demethylase-1 (LSD1) depletion disrupts monogenic and monoallelic odorant receptor (OR) expression in an olfactory neuronal cell line. Mol Cell Neurosci, 82, 1-11. doi:10.1016/j.mcn.2017.04.005

Wong, X., Luperchio, T. R., \& Reddy, K. L. (2014). NET gains and losses: the role of changing nuclear envelope proteomes in genome regulation. Curr Opin Cell Biol, 28, 105-120. doi:10.1016/j.ceb.2014.04.005

Ye, Q., \& Worman, H. J. (1996). Interaction between an integral protein of the nuclear envelope inner membrane and human chromodomain proteins homologous to Drosophila HP1. J Biol Chem, 271(25), 14653-14656. doi:10.1074/jbc.271.25.14653

\section{Figure legends}

Figure 1. Kdm4b initiate Or59b expression. Whole-mount brain staining shows the expression of GFP (green) driven by the Or59b reporter. Synaptic neuropil regions are labeled with the presynaptic marker nc82 (magenta). Below each merged image, the GFP channel is shown. The marked region defines the antennal lobes and the DM4 glomerulus. (A) Loss of expression of the Or59b reporter is observed in knockdown of Kdm4b but not Kdm4a. Control flies were crossed to w1118. (B) Knock down of Kdm4b after the initiation of OR expression does not affect the expression of Or59b reporter.

Figure 2. dLsd1 stabilize OR expression. Whole-mount brain staining shows the expression of GFP (green) driven by the OR reporter(s). Synaptic neuropil regions are labeled with the presynaptic marker nc82 (magenta). Below each merged image, the GFP channel is shown. AL and labeled glomeruli a are marked. (A) Or59b reporter expression is abolished in dLsd1 knockdown flies. Control flies 
were crossed to $\mathrm{w}^{1118}$. (B) Or92a and Or47b reporter GFP expression label the VA2 and VA1v glomeruli. Note the loss of Or92a reporter GFP expression in VA2 but not by the Or47b reporter (VA1v) in dLsd1 knock down flies. (C) GFP expression (green) driven by an Or59b minimal enhancer in su(var)3-906 and $\mathrm{dLsd}^{09}$ heterozygote flies. (D) The disturbed expression of the minimal enhancer in heterozygote flies is rescued double heterozygotes for su(var)3-906 and dLsd $1^{09}$. (E) Knock down of dLsd1 after the initiation of OR expression does not affect the expression of Or59b reporter but hampers the expression of the Or59b minimal enhancer.

Figure 3. Lbr and HP1b defines the state of adult OR expression. Whole-mount brain staining presynaptic marker nc82 (magenta). The antennal lobe and the DM4 glomerulus are out lined in the GFP channel. GFP expression (green) driven by the Or59bME reporter (A ) and the Or59b reporter (B) is abolished in Lbr and HP1b knockdown flies. Control flies were crossed to $\mathrm{w}^{1118}$.

Figure 4. Or59b expression matures and dLsd1, Su(var)3-9and Kdm4b show dynamic expression in

early-life Drosophila. (A) Whole-mount brain and antenna staining shows the expression of GFP (green) driven by the $O r 59 b$ reporter. Synaptic neuropil regions are labeled with the presynaptic marker nc82 (magenta). Below each merged image, the GFP expression in the antenna is shown as the white channel. DPE, days post eclosion. (B) qPCR analysis of dLsd1mRNA levels in antenna, 1 hour and 7 hours after eclosion. Note that the expression levels increase to almost twice in the first seven hours of adult fly's life. (D) The mRNA levels of the $\mathrm{Kdm} 4 \mathrm{~b}$ in the antenna was measured by qPCR and compared between DPE 0 and 7. 
510 Note that unlike $\mathrm{Su}($ var)3-9 and dLsd1, Kdm4b mRNA levels decrease post eclosion. (E) qPCR analysis of $\mathrm{Su}(\mathrm{var}) 3-9$ and dLsdl expression level in the brain at DPE 0, 3 and 7. Note that only dLsd 1 increase in the brain post eclosion.

Figure 5. Environment changes during and after the critical period have contrasting effects on OR gene regulation, dLsd1 and Su(var)3-9expression

516 (A) Diagram decapitating the time course of experiments and low temperature shifts. Each box represents 24 hours. Low temperature exposure time is presented as dark blue boxes. (B) qPCR analysis of su(var)3-9and dLsd1 expression in antenna after low temperature shifts at DPE 1 or $7 .(* \mathrm{p}<0.05 ; * * \mathrm{p}<0.01 ; * * * \mathrm{p}<0.001$; error bars represent SEM). (C) The percentage fractions of $O r 59 \mathrm{bME}-G F P$ brains that show control, ectopic or loss of expression after exposure to low temperature at DPE 1,2,3 or 7. (D) Diagram describing the restricted low temperature shifts. (E) The percentage fractions of 59bME reporter brains that showed control (DM4), ectopic or loss of GFP expression after the low temperature shifts denoted in

D.

Figure 6. Environmental stress during the critical period induce permanent changes in OSN gene regulation (A) The percentage fractions of the brains showing control or ectopic expression of Or59bME-GFP in the brain after exposure to low temperature at the day of eclosion for a period of three days. Note that neither a recovery of 18 days at $24^{\circ} \mathrm{C}$ nor a second exposure to low temperature from day 7 after eclosion affect the expression phenotype of the minimal enhancer. (B) The percentage fractions of the brains showing control, ectopic or loss of Or59bME reporter GFP expression in the brain after exposure to low temperature four days after eclosion for a period of three days. Note that a period of 14 days at $24^{\circ} \mathrm{C}$ recovers the phenotype to single OSN class expression. 
bioRxiv preprint doi: https://doi.org/10.1101/2020.02.12.945428; this version posted February 12, 2020. The copyright holder for this preprint (which was not certified by peer review) is the author/funder, who has granted bioRxiv a license to display the preprint in perpetuity. It is made available under aCC-BY 4.0 International license.

535

536

537

538

539

540

541

542

543

544

545

546

547

548

549 

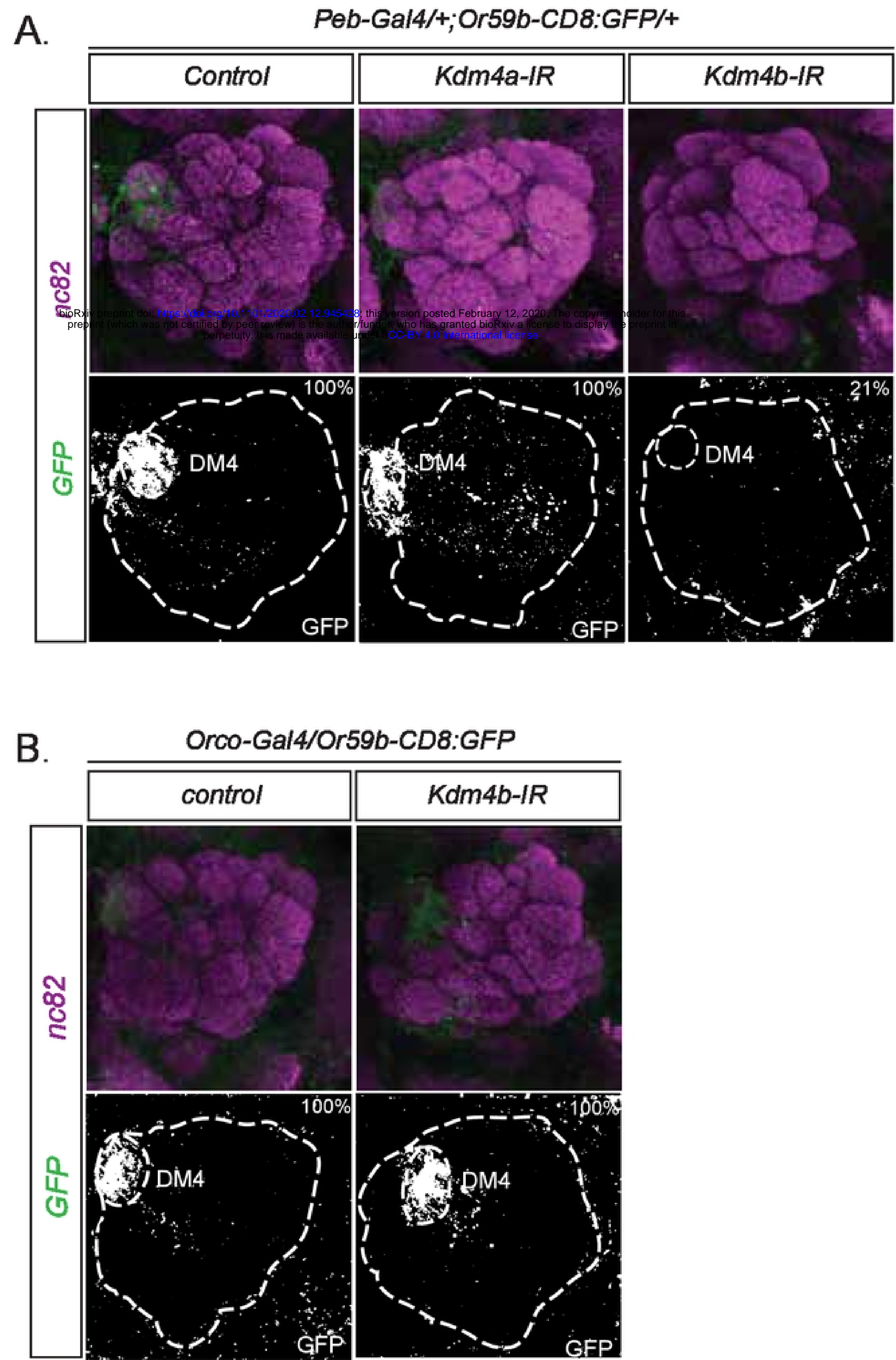

Figure 1 
A.

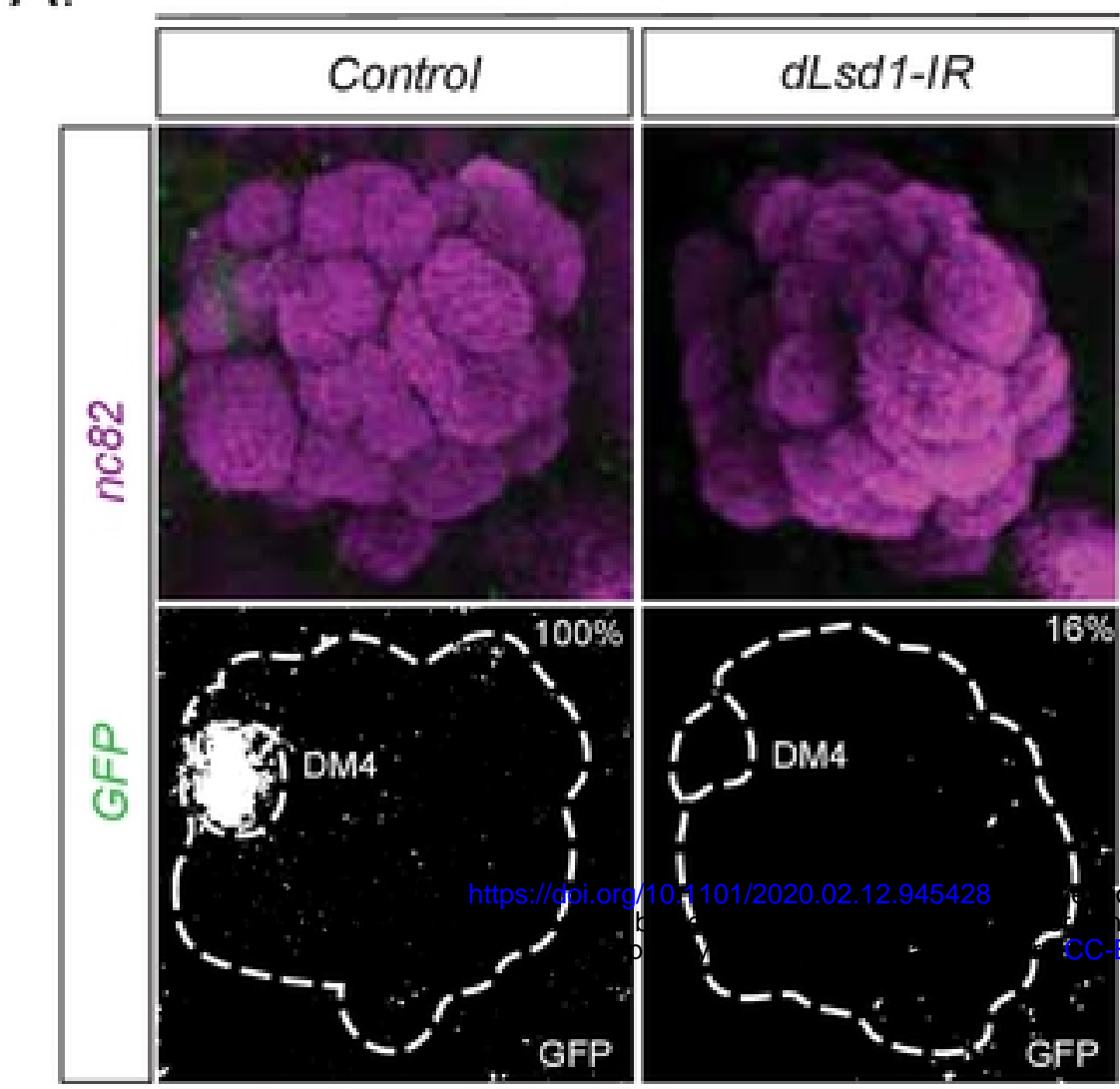

B. Peb-Gal4;Or92a-CD8:GFP,Or47b-CD8:GFP/+

on posted February 12,2020 . The copyrig

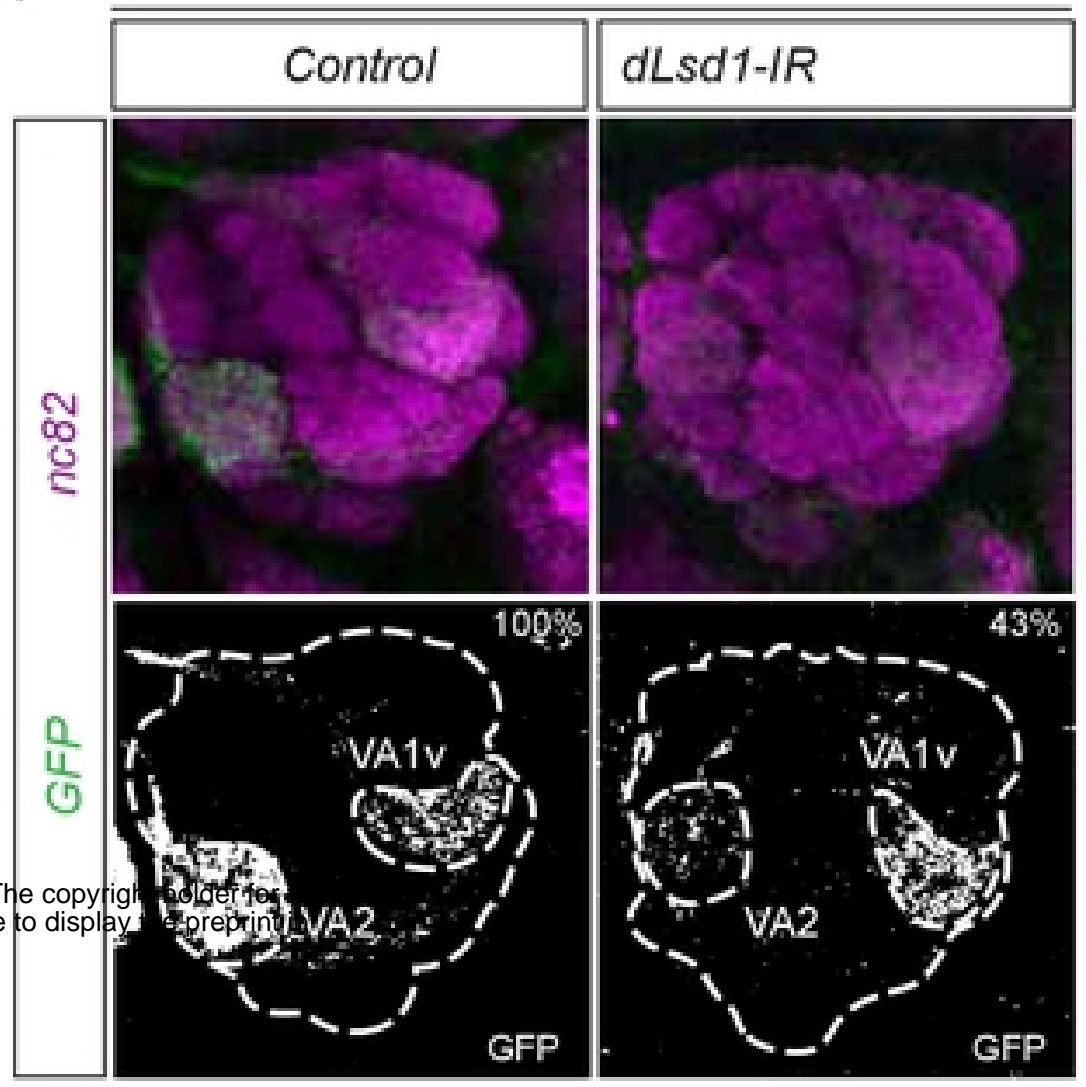

C.
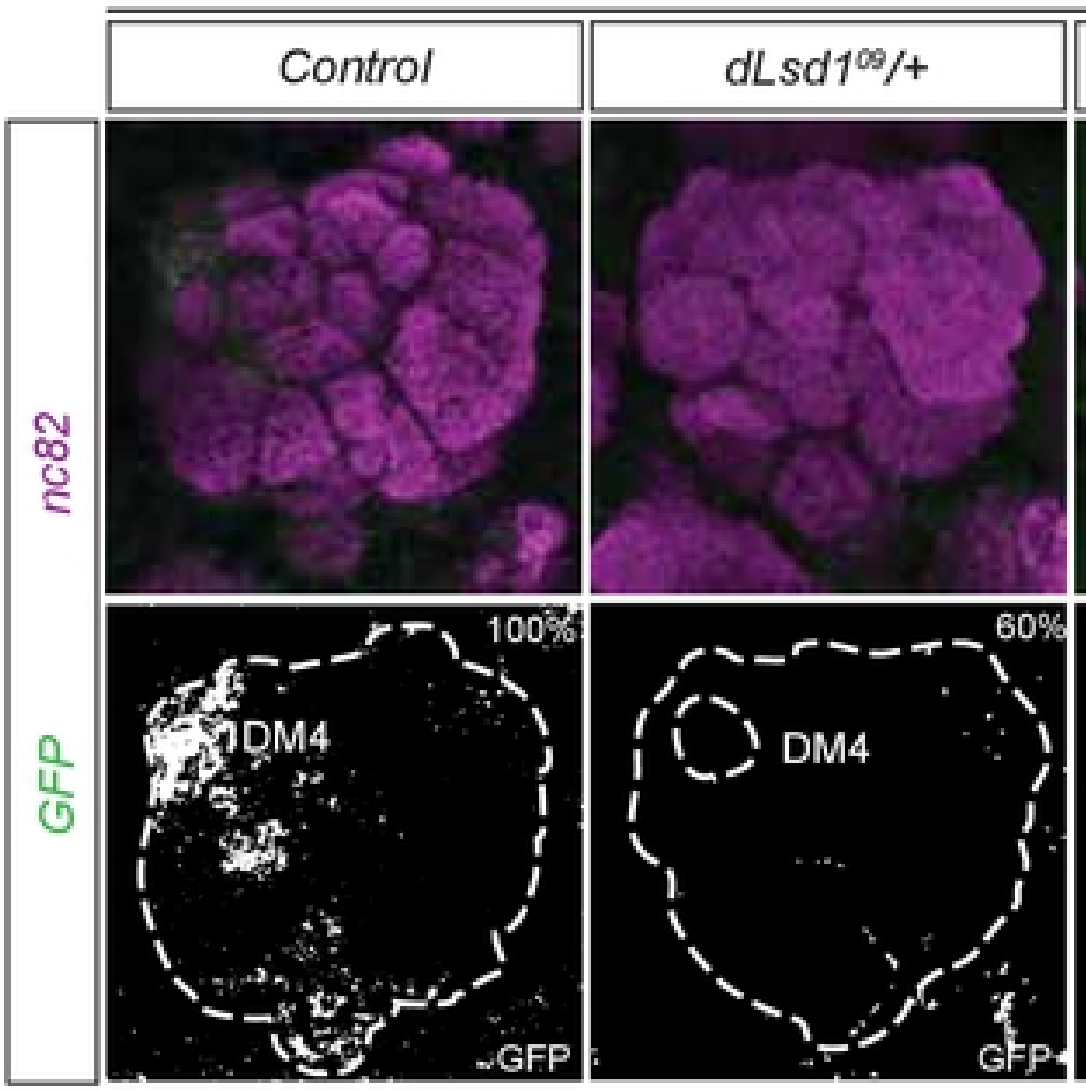

Or59bME-CD8:GFP/+

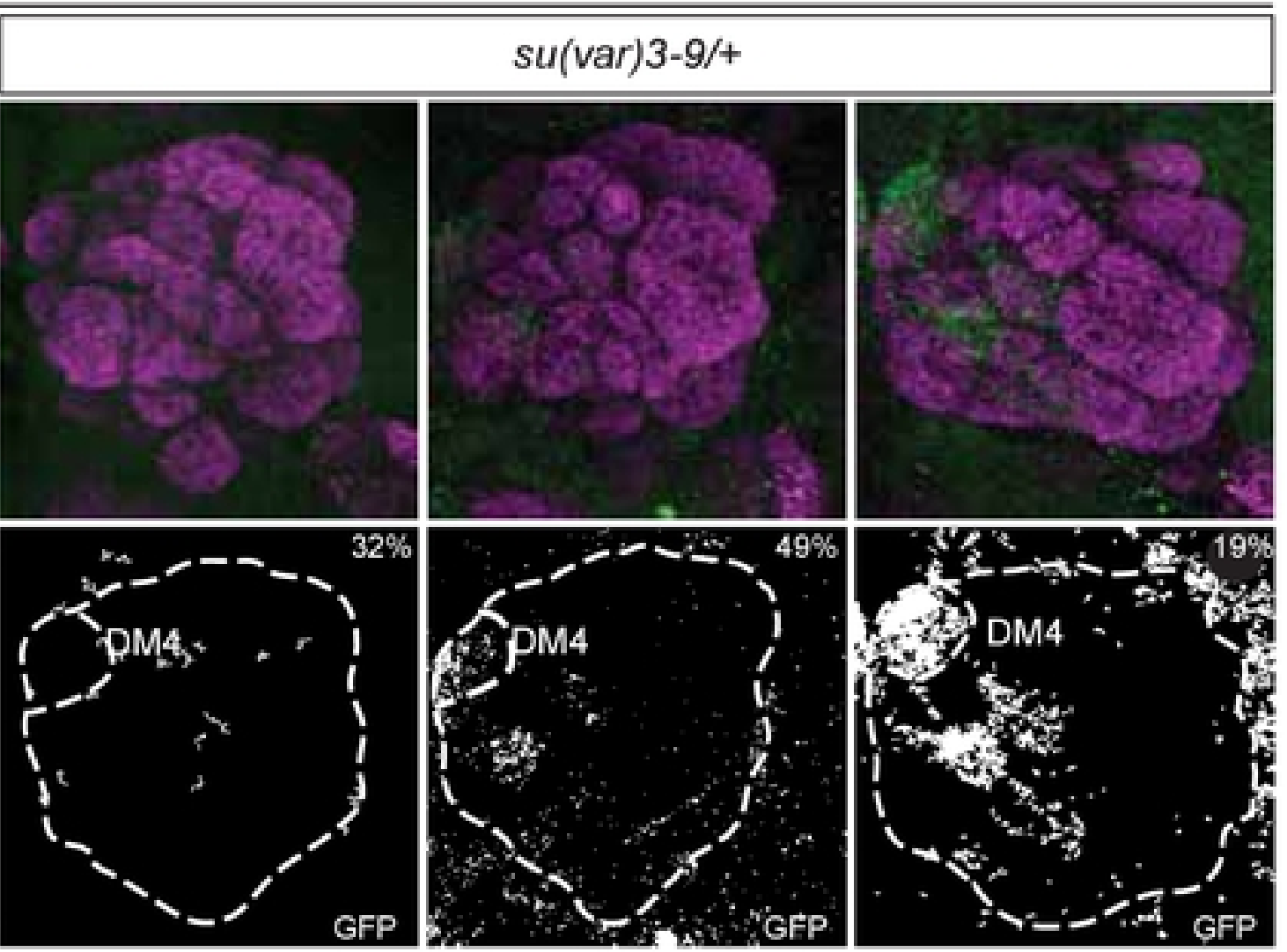

D

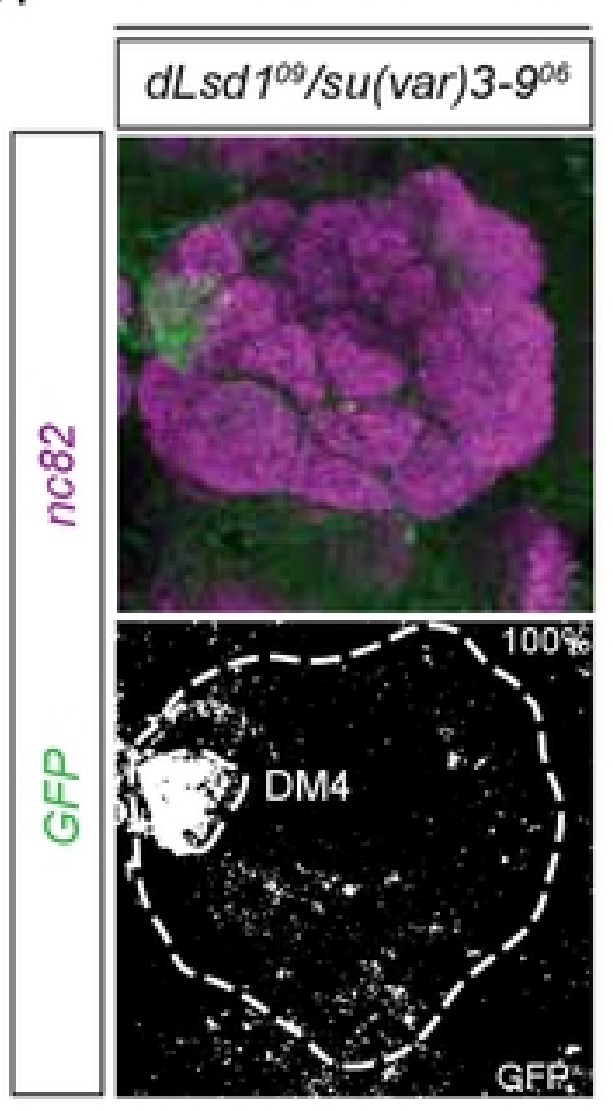

E. Orco-Gal4,Or59bME-CD8:GFP/+ Orco-Gal4,Or59b-CD8:GFP /+

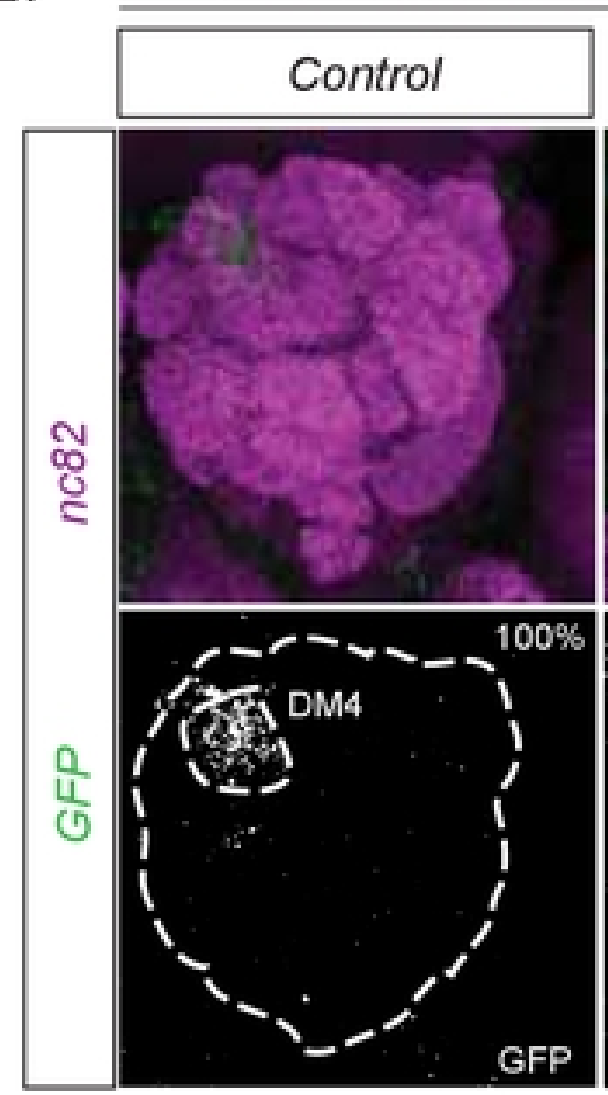

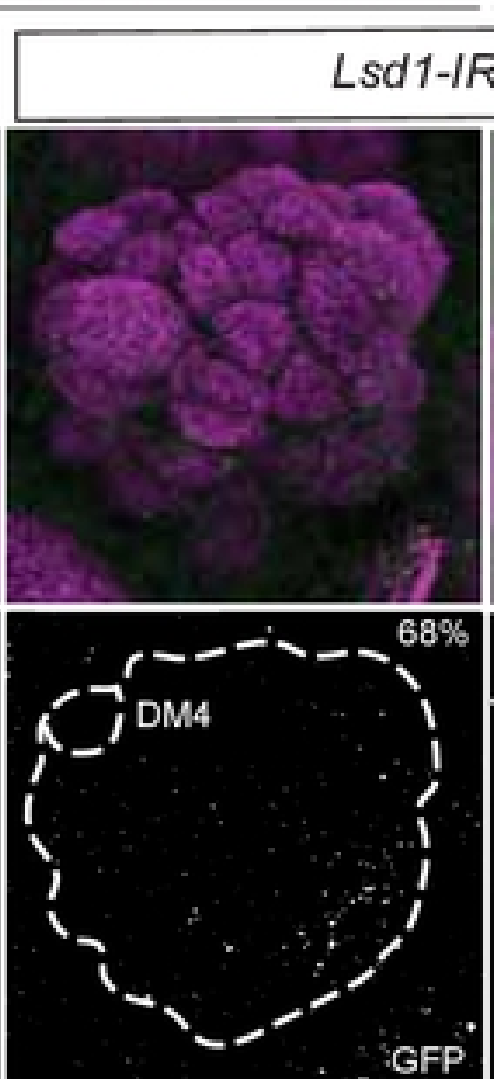

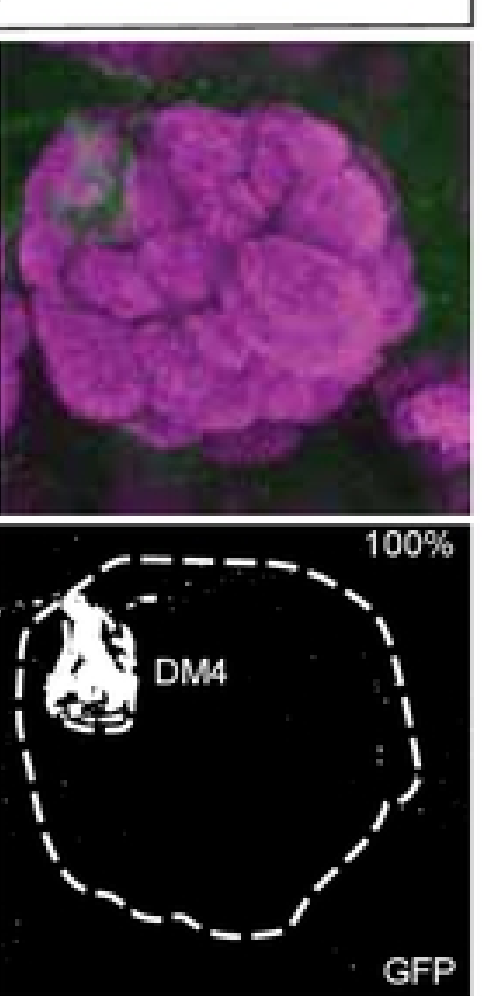

Figure 2 
A.

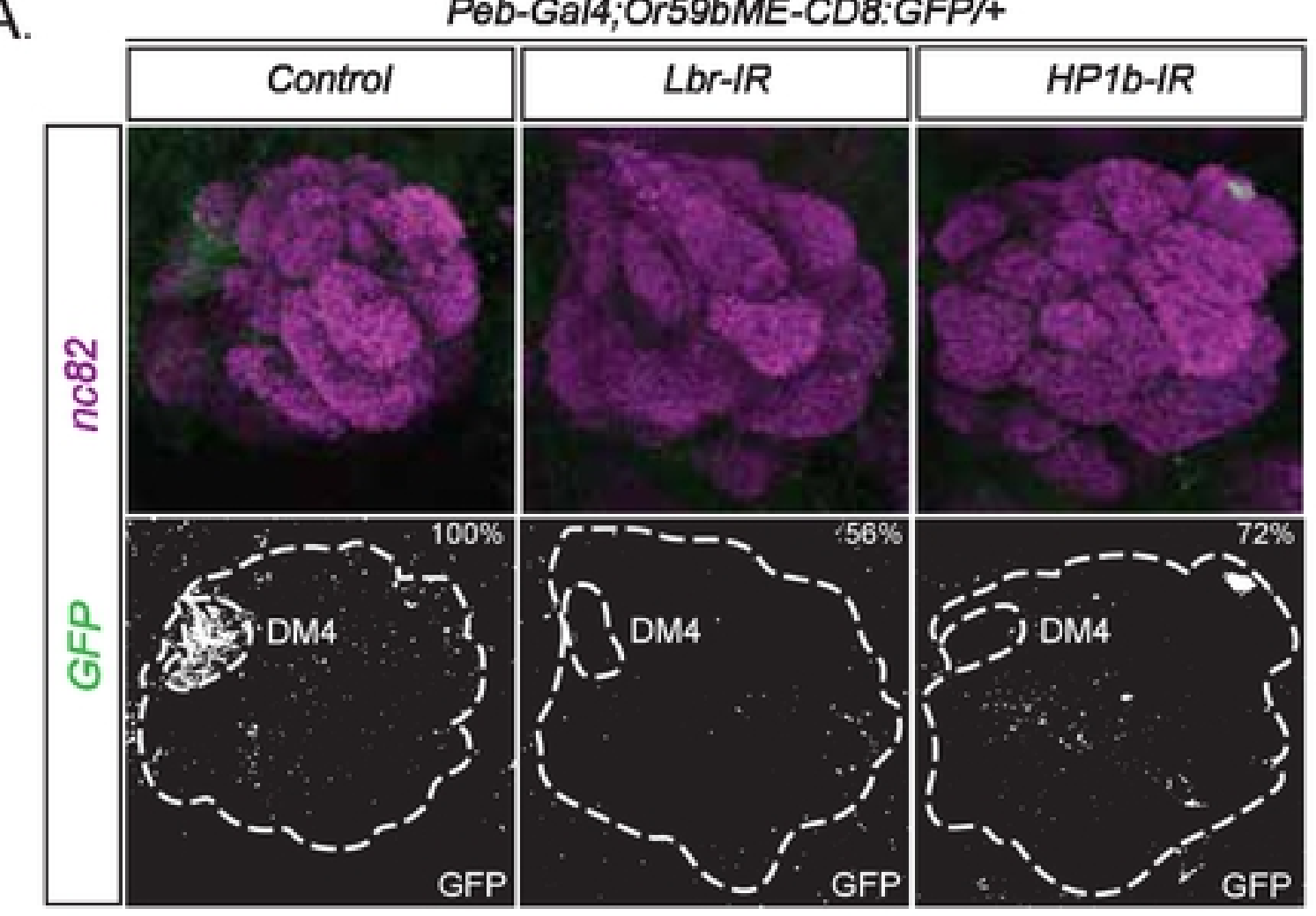

B.

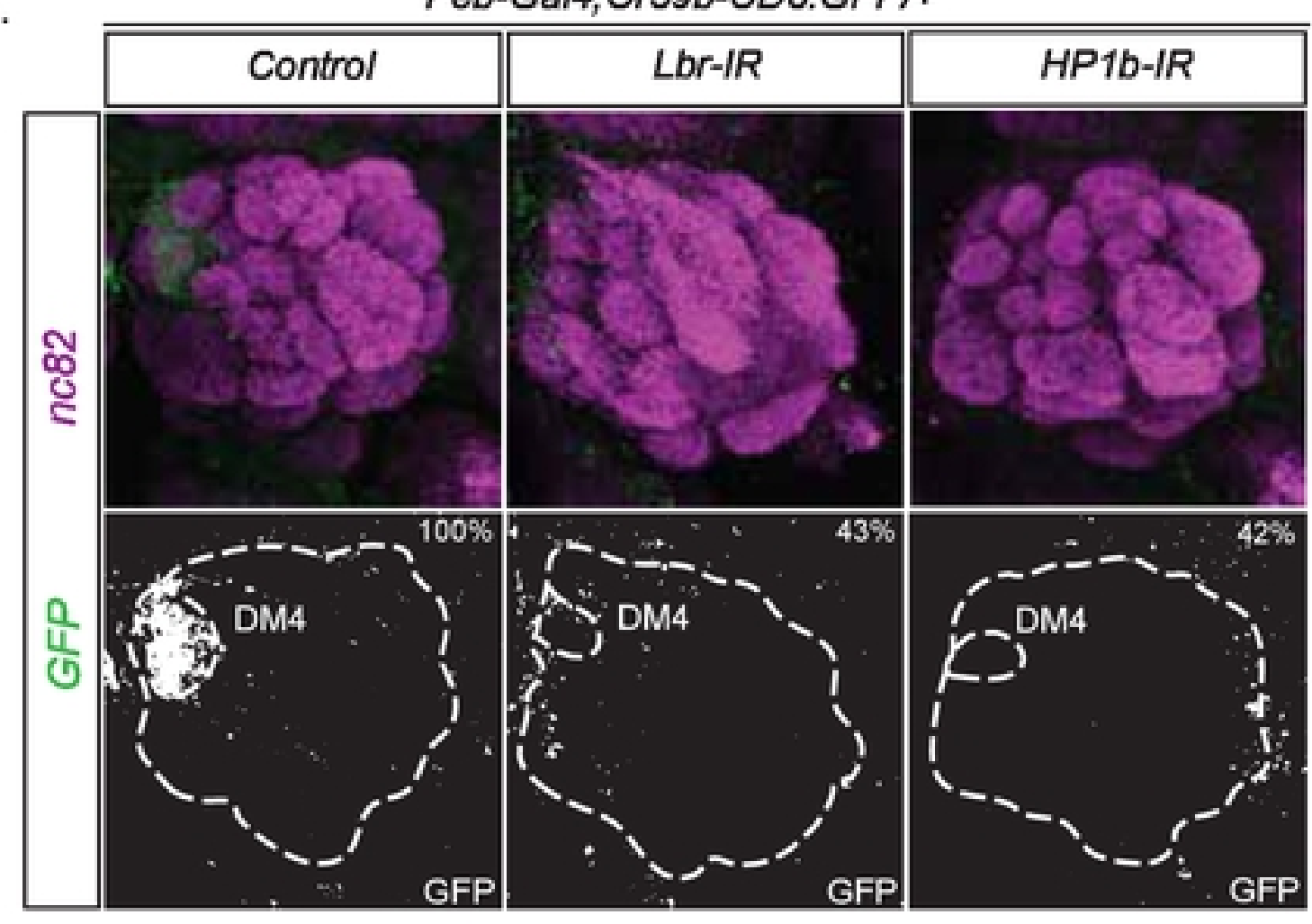

Figure 3 


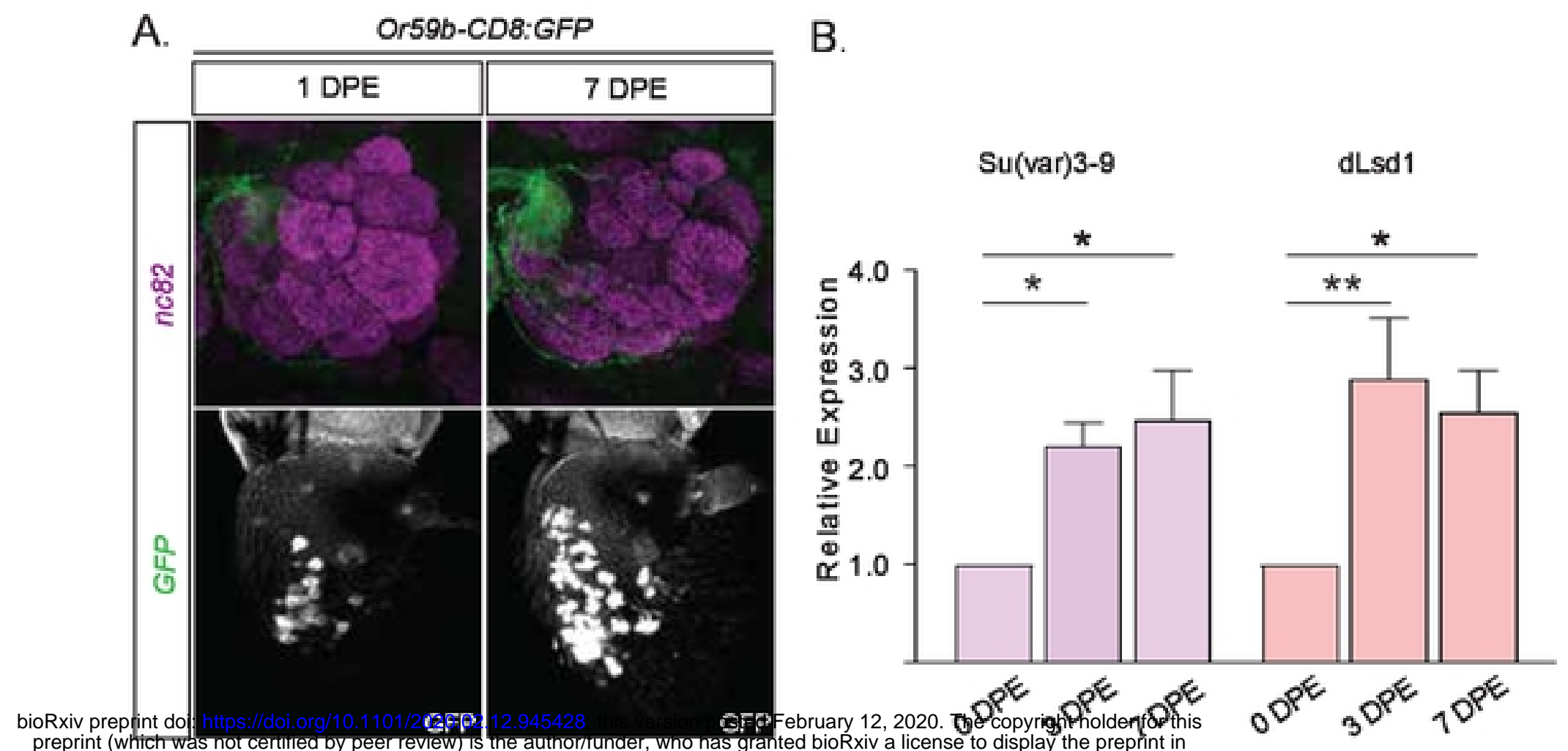

C.

Su(var)3-9

dLsd1

D.

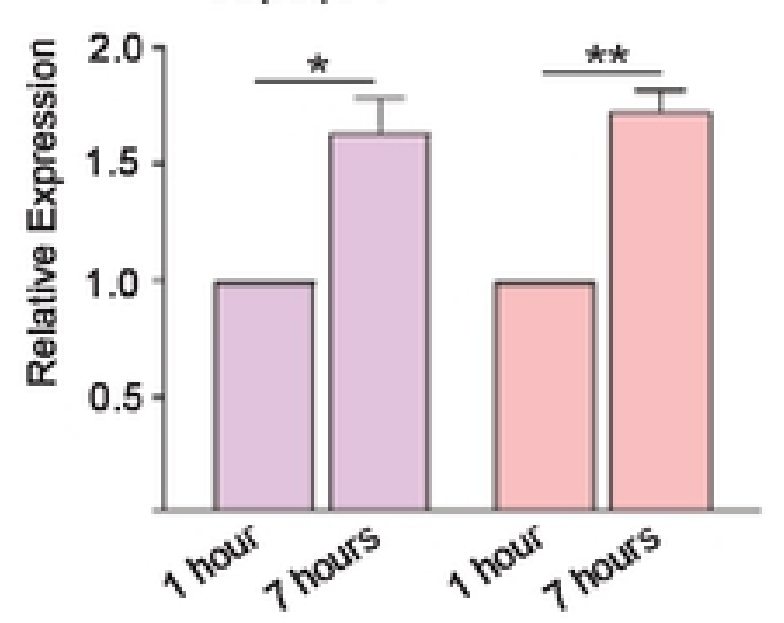

$\mathrm{Kdm} 4 \mathrm{~b}$

E.
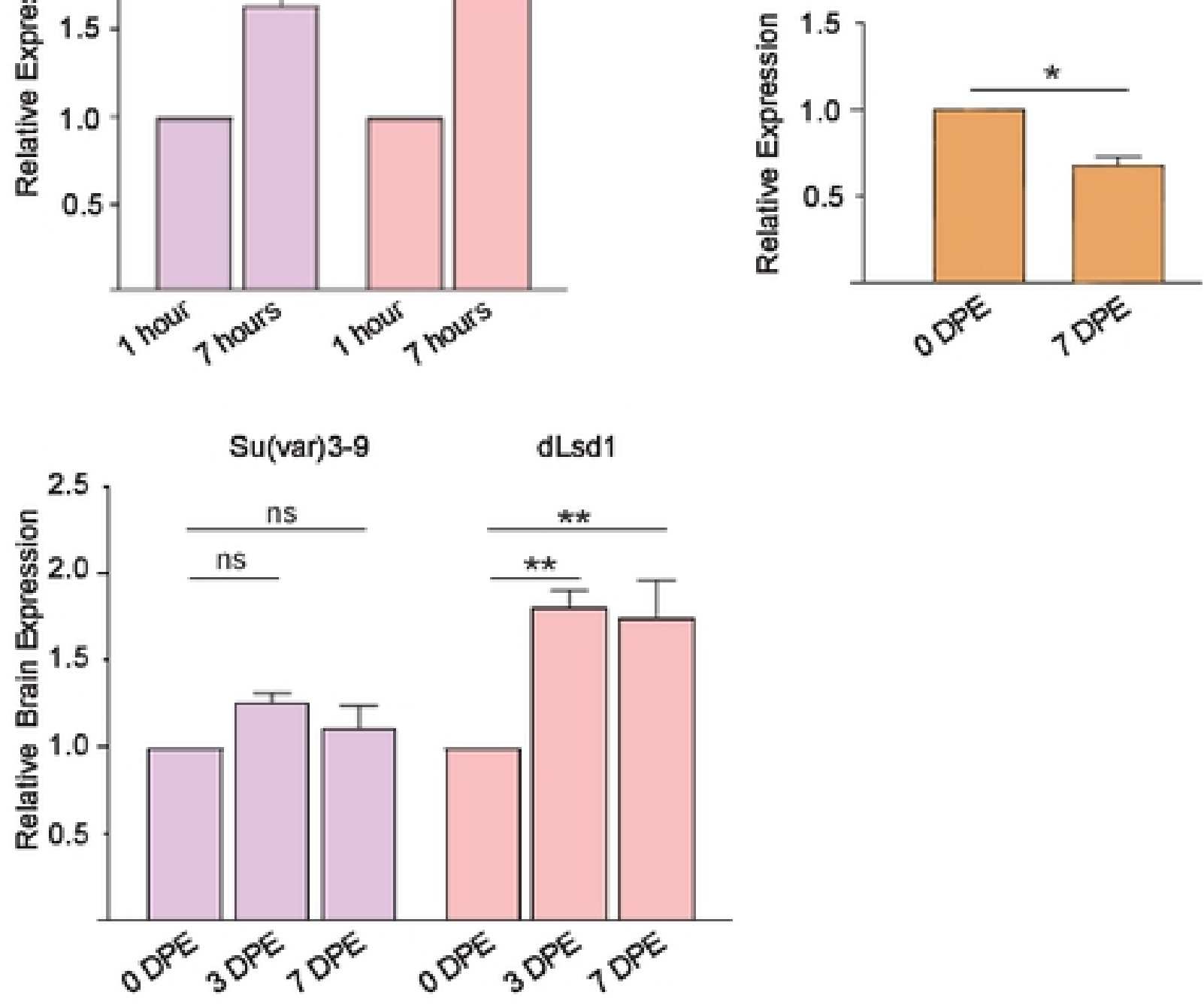

Figure 4 
A

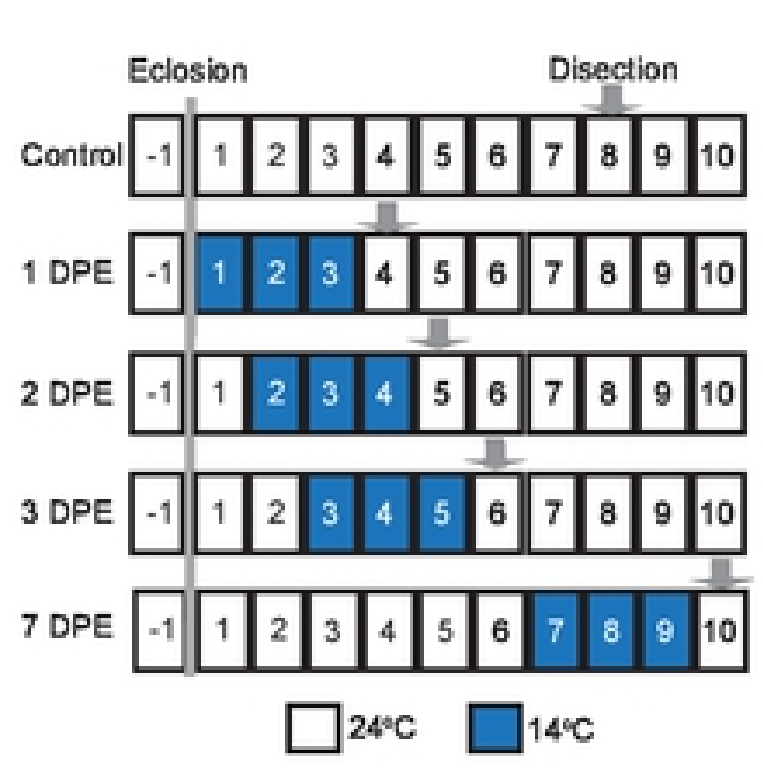

B.

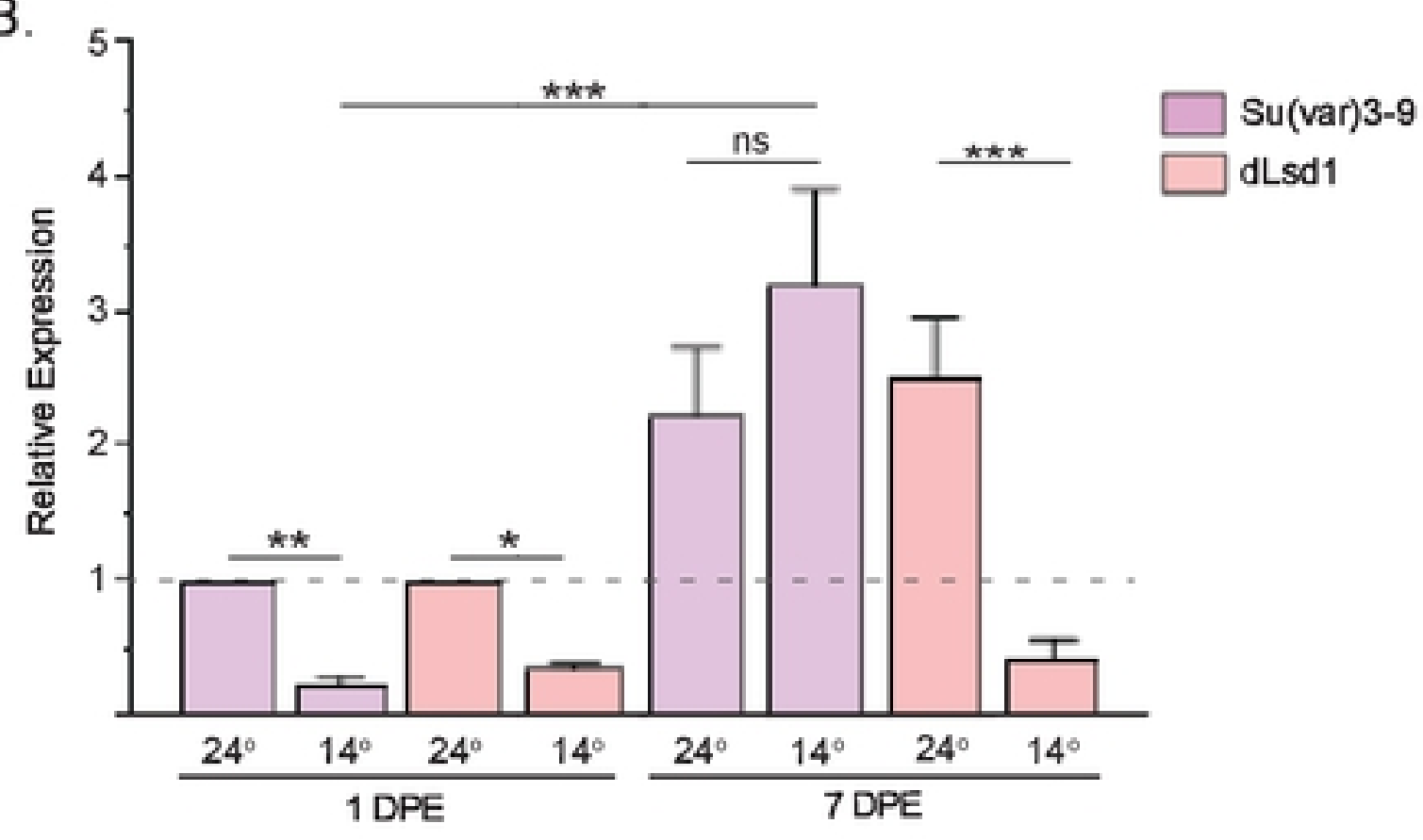

C.

$\mathrm{n}=21 \quad \mathrm{n}=16 \quad \mathrm{n}=17 \quad \mathrm{n}=\mathbf{2 3} \quad \mathrm{n}=18$

D.

bioRxiv pilep@ hी(doi: https://doi.org/10.1101/2020.02.12.945428; this version posted February 12, 2020. The copyright holder for this
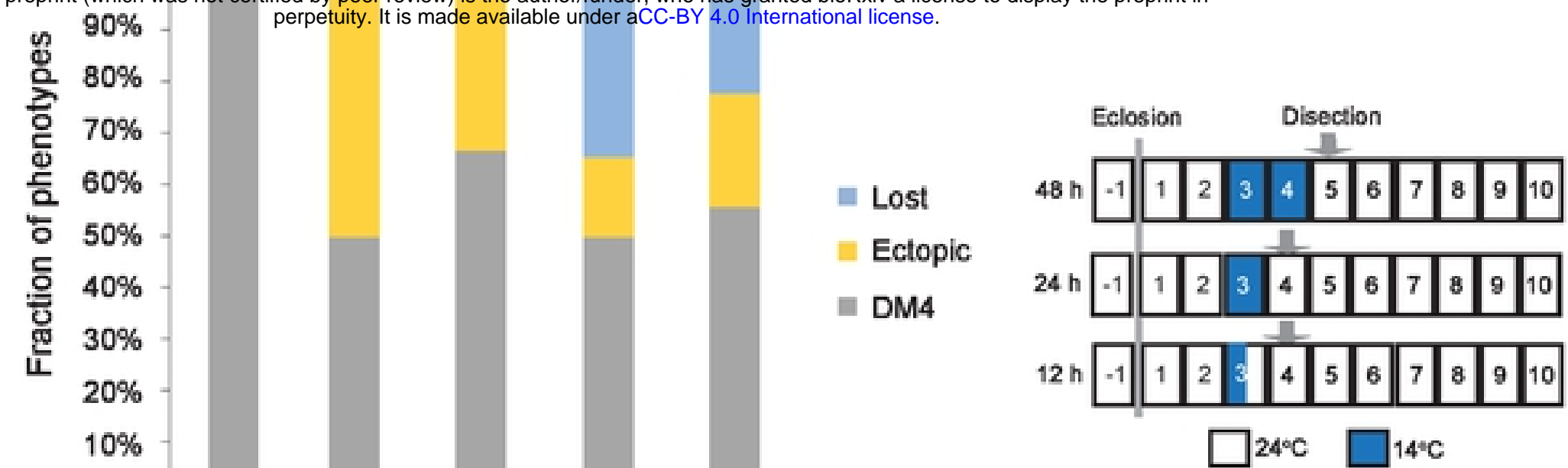

$\square^{24^{\circ} \mathrm{C}} \square 14^{\circ} \mathrm{C}$

E.

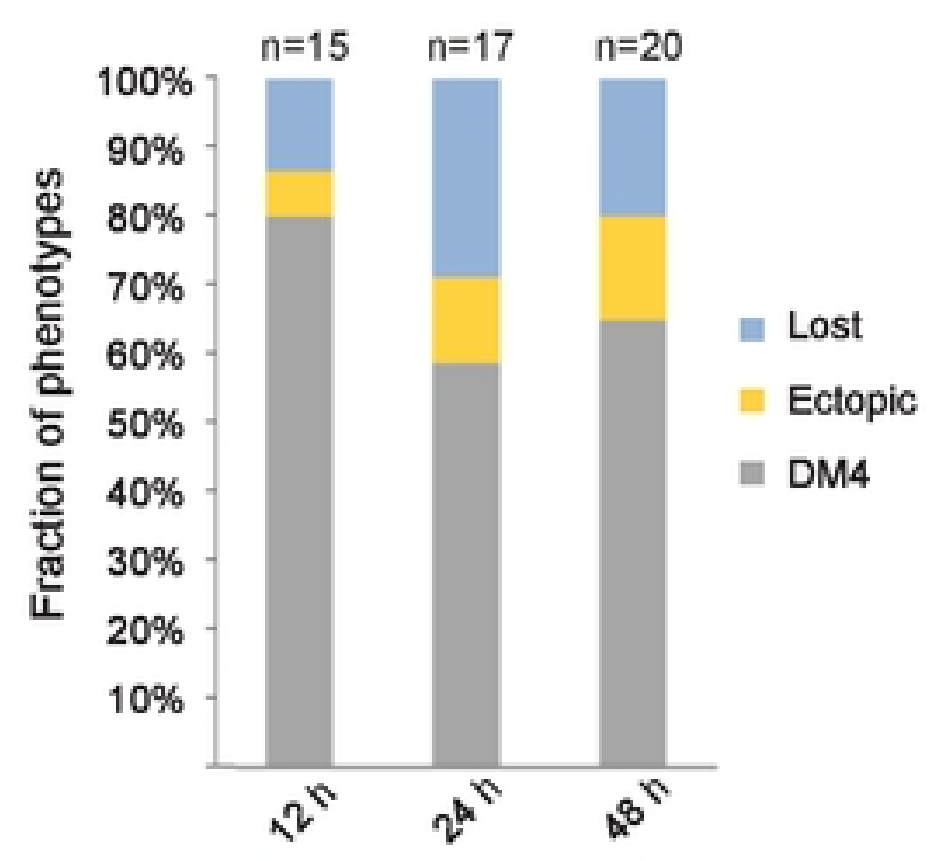

Figure 5 
A.

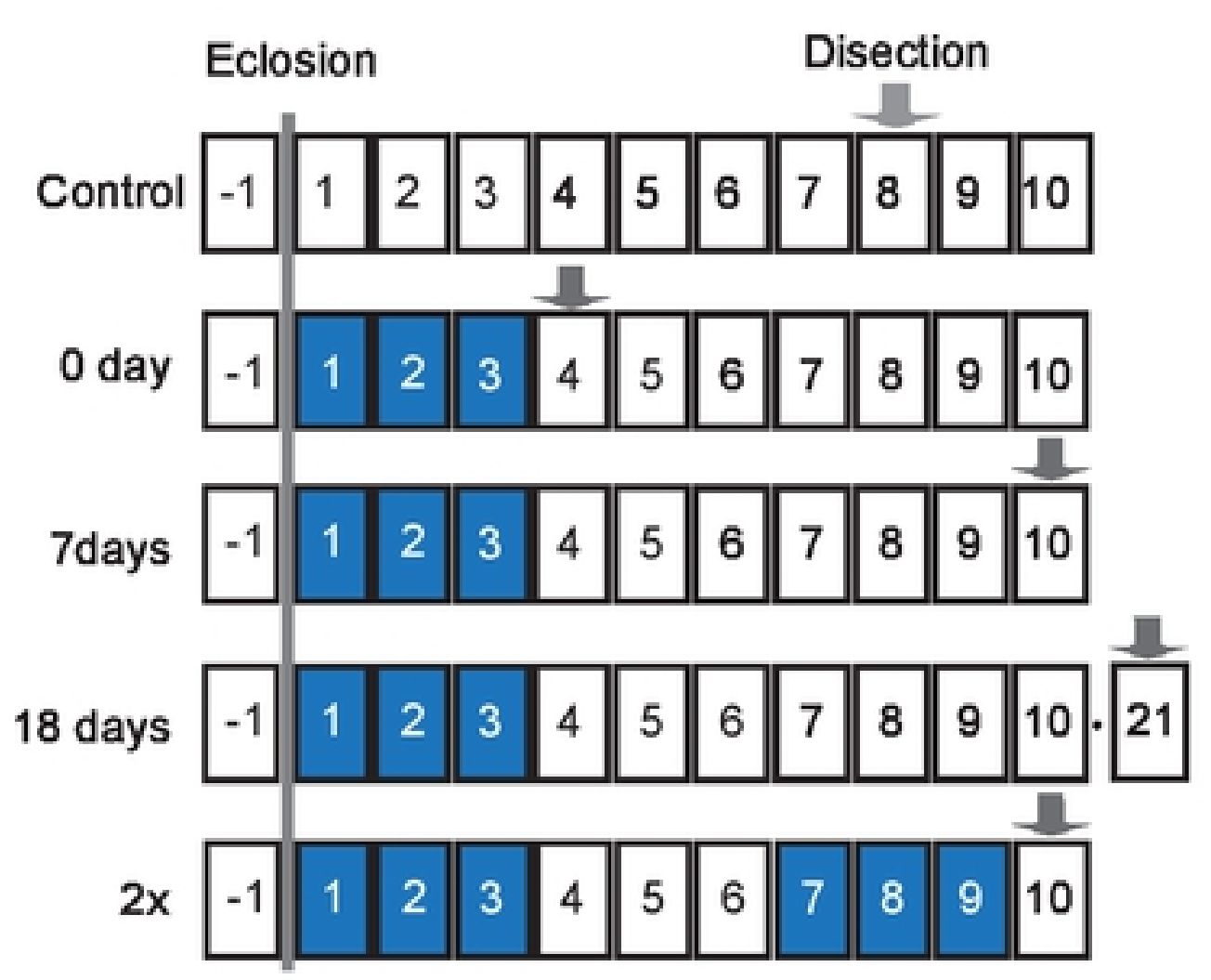

$\square 24^{\circ} \mathrm{C} \quad \square 14^{\circ} \mathrm{C}$

B.

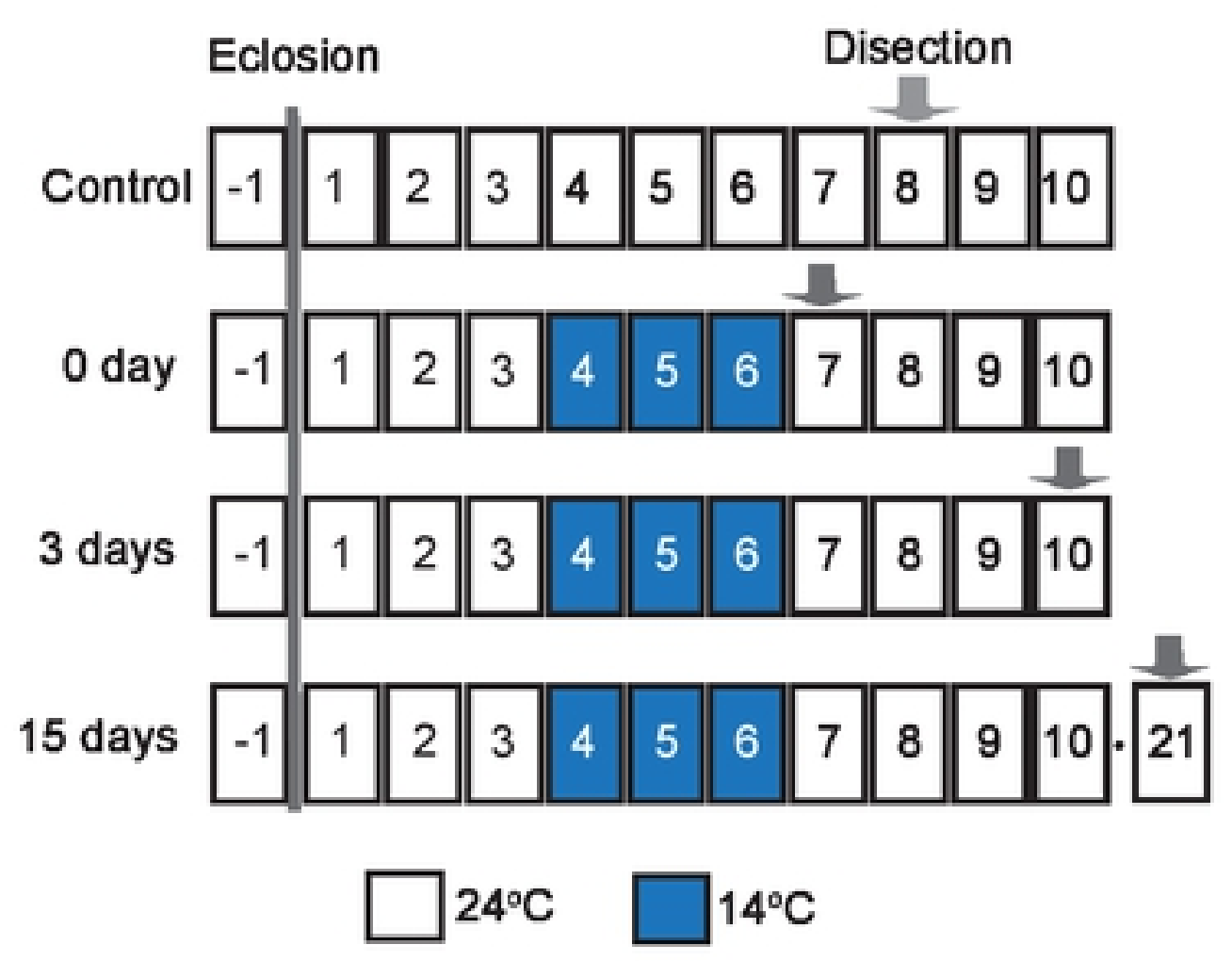

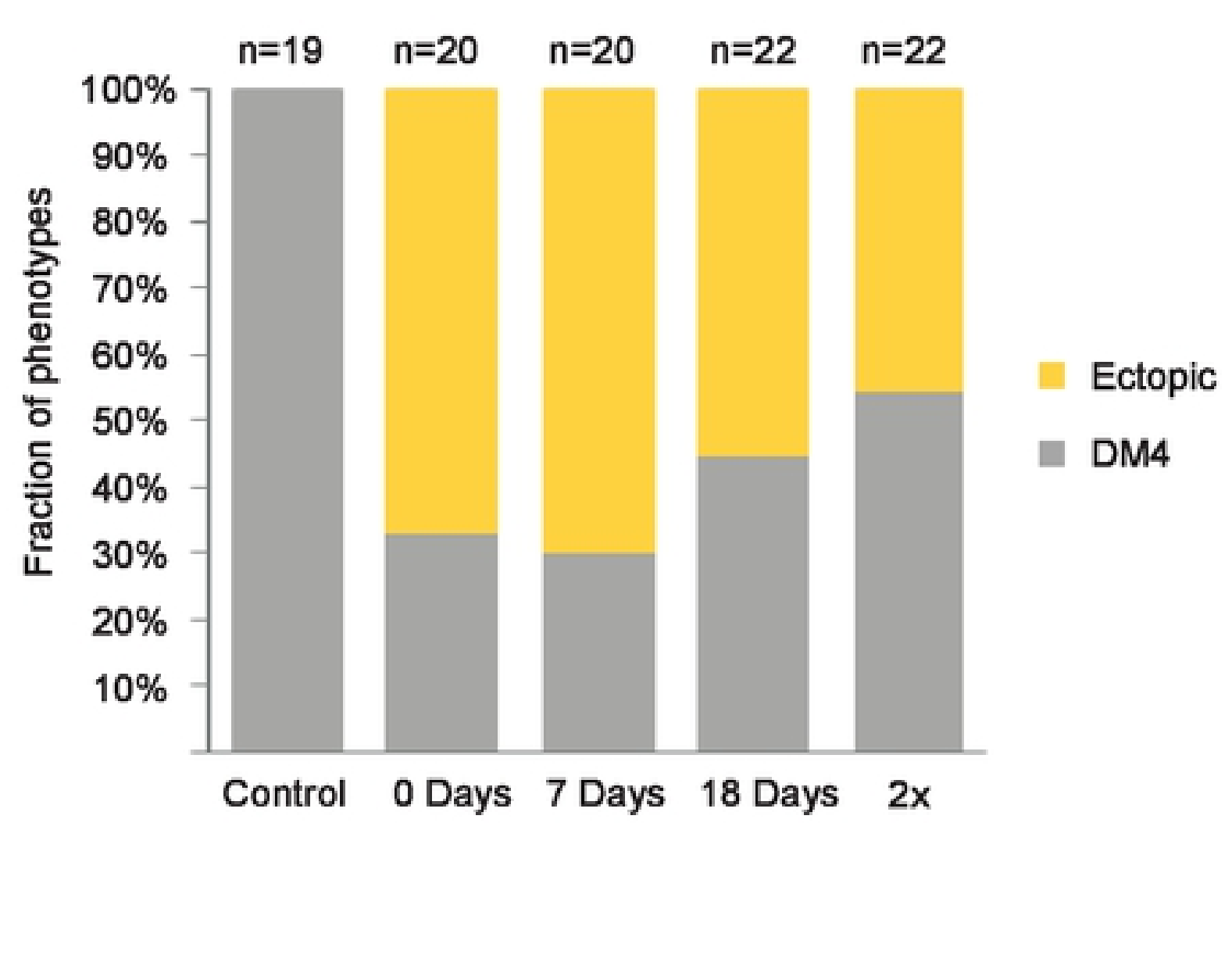

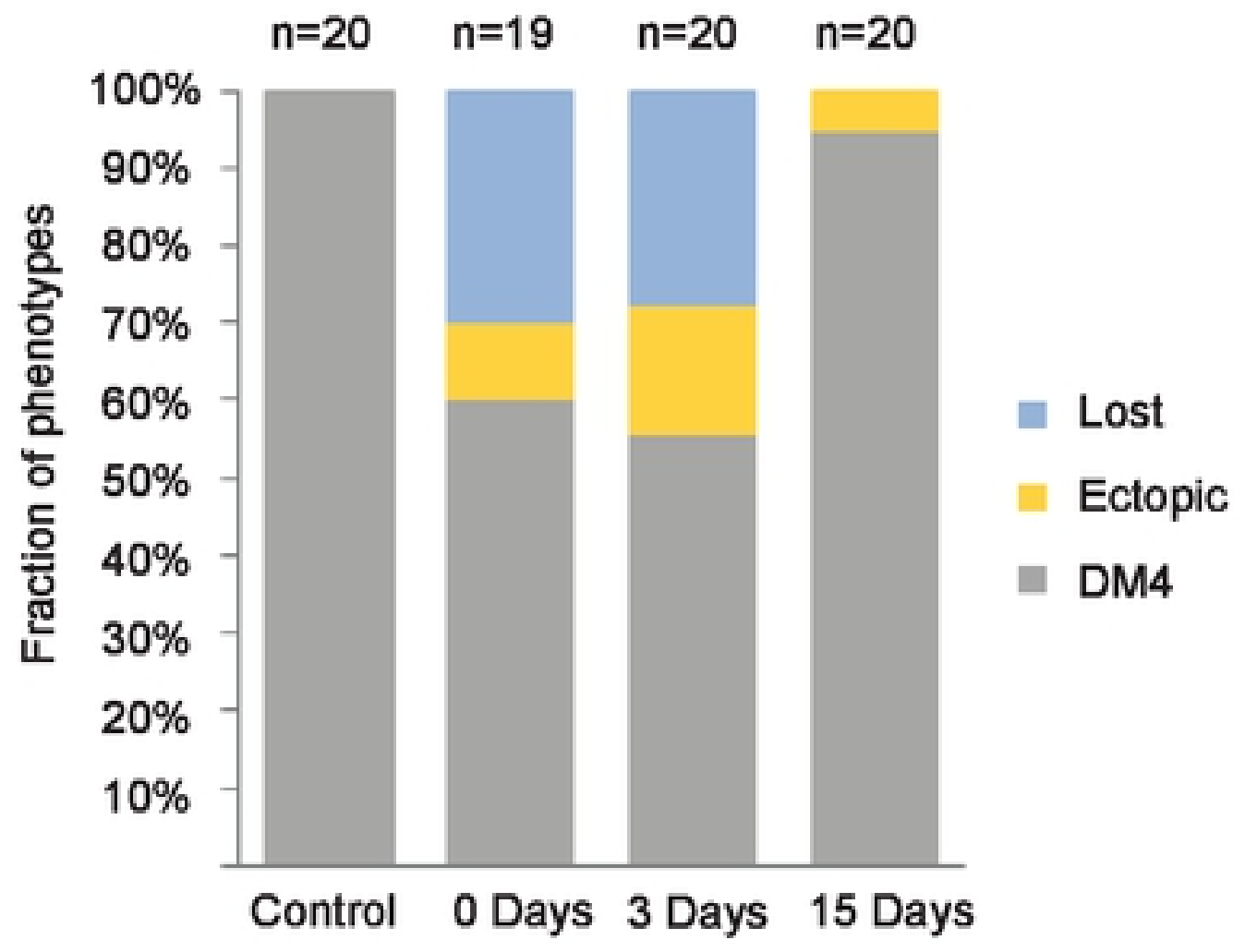

Figure 6 